\title{
Théorie de la génération de la houle cylindrique par un batteur plan
}

( $2^{\circ}$ ORDRE D'APPROXIMATION)

\section{Theory of the generation of a cylindrical wave by a straight-fronted wave generator}

(Second order of approximation)

\author{
PAR PLERE FONTANET

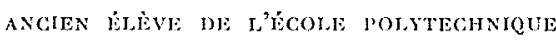

(Suite et fin) ${ }^{(*)}$

\begin{abstract}
La lhéorie du mollnement engendré par un batfeur plan dans un canal indéfini, de profondeur et de largeur constantes, rempli d'un fluide parfait, pesant et incompressible, a été faite au premier ordre d'approximation par MM. Havelock, Biesel et Kravtchenlo. Le présent travail est consacré à l'étude de la deuxième approximation, ainsi qu'au calcul de l'harmonique non linéaire émis par le volet. Les abaques relatifs au batteur piston ont été tracés et reproduits dans le texte.

La solution se présente sous la forme d'une somme de séries uniformément convergentes dans toute la masse du fluide, ainsi que sur les frontières, sauf aux extrémités du volet, où la solution possède des singularités logarithmiques.
\end{abstract}

The theory of the movement produced by a straight-fronted wave generator in an indefinite canal of constant depth and breadth and containing a perfect ponderable and incompressible fluid, has been established to a first order of approximation by Messrs. Havelock, Biesel and Kravtchenko. The present work is concerned with the study of a second order of approximation and the calculation of the non-linear harmonic emitted by the flap. Graphs for a plunger-type wave generator have been plotted and are reproduced in the text.

The solution takes the form of a sum of uniformly converging series throughout the fluid mass and at its boundaries, except at the flap ends, where logarithmic singularities occur.

\section{CHAPITRE IV}

\section{CALCUL DE L'HARMONIQUE ET DE L'OSCILlATION LOCALE DE PERIODE T/2}

Les équations et conditions aux limites auxquelles doit satisfaire la fonction $\mathrm{J}_{2}\left(x_{0}, y_{0}, t\right)$ étant linéaires, on peut décomposer cette dernière en trois fonctions, qui vérifient sur le batteur chacune des conditions aux limites suivantes:

$$
\frac{\partial \mathrm{J}_{2}^{\prime}}{\partial x_{0}}=\mathrm{F}_{1}\left(y_{0}\right) \quad \frac{\partial \mathrm{J}_{2}^{\prime \prime}}{\partial x_{0}}=\mathrm{F}_{2}\left(y_{0}\right) \sin 2 k t \quad \frac{\partial \mathrm{J}^{\prime \prime \prime} 2}{\partial x_{0}}=\mathrm{F}_{3}\left(y_{0}\right) \cos 2 k t
$$

$1^{\circ}$ Recherche de la fonction $\mathrm{J}_{2}^{\prime}\left(x_{0}, y_{0}\right)$ :

La fonction $\mathrm{J}_{2}^{\prime}\left(x_{0}, y_{0}\right)$ ne dépend pas du temps. La condition de surface se réduit donc à :

(') Cf. La Houille Blanche, $\mathrm{n}^{\circ} 1-1961$, page 3 . 


$$
\frac{\partial \mathrm{J}_{2}^{\prime}}{\partial y_{0}}=0
$$

Les solutions à variables séparées de l'équation $\Delta \mathbf{J}_{2}^{\prime}=0$, vérifiant les conditions de fonds et de surface ainsi que les conditions à l'infini, appartiennent à un type unique :

avec :

$$
J_{2}^{\prime}\left(x_{0}, y_{0}\right)=\sum_{1}^{\infty} \frac{c_{r}^{\prime}}{e^{2}} e-\mu_{n} x_{0} \cos \mu_{n} y_{11}
$$

$$
\mu_{r} h=\pi+r \pi
$$

Ce terme indépendant du temps représente une variation du niveau moyen qui devient négligeable à faible distance du batteur. On choisira les coefficients $A_{p}$ de manière à satisfaire à la condition limite sur le volet, soit :

$$
\frac{\partial \mathrm{J}_{2}^{\prime}}{\partial x_{0}}=\cdots-\sum_{1}^{\infty} u_{r} \frac{c_{r}^{\prime}}{e^{2}} \cos u_{r} y_{0}=\mathrm{F}_{1}\left(y_{0}\right)
$$

La suite des fonctions $\cos \mu_{r} y_{0}$ est complète et orthogonale, puisque ce sont les fonctions propres de l'équation différentielle $U^{\prime \prime}+\mu . U=0$ assujetties aux conditions limites :

$$
\mathrm{U}^{\prime}(0)=\mathrm{U}^{\prime}(h)=0 .
$$

Par suite, ce développement est possible et l'on a :

$$
\frac{c_{r}^{\prime}}{e^{2}}=-\frac{-\int_{0}^{h} F_{1}\left(y_{0}\right) \cos \mu_{r,} y_{0} d y_{0}}{\mu_{r . r}^{h} \int_{0}^{h} \cos ^{2} \mu_{r}, y_{0} d y_{0}}=\frac{-2 \int_{0}^{h} \mathrm{~F}_{\mathrm{I}}\left(y_{0}\right) \cos \mu_{r} y_{0} d y_{0}}{\mu_{r}, h+\sin \mu_{r} h \cos \mu_{r} h}
$$

$2^{\circ}$ Recherche de la Fonction $\mathrm{J}_{2}^{\prime \prime}\left(x_{0}, y_{0}, t\right)$ :

On forme avec les solutions à variables séparées une combinaison linéaire qui vérifie les conditions 1,2 et 4 :

$$
J^{\prime \prime}, 2\left(x_{0}, y_{0}, t\right)=\frac{a_{0}^{\prime}}{m_{0}^{\prime} e^{2}} \frac{\operatorname{ch~} m_{0}^{\prime} y_{0}}{\operatorname{sh~} m_{0}^{\prime}{ }_{0} h} \cos \left(2 k t-m_{0}^{\prime} x_{0}\right)+\sum_{1}^{\infty} \frac{2 \alpha_{r}^{\prime}}{m_{r}^{\prime} e^{2}} e^{-i m m_{r}^{\prime} x_{0}} \frac{\operatorname{ch} m_{r}^{\prime} y_{0}}{\operatorname{sh} m_{r}^{\prime} h} \sin 2 k t
$$

$m_{0}^{\prime}$ étant l'unique racine réelle et $m^{\prime}$. l'une des racines imaginaires pures de $4 k^{2}=m^{\prime} g$ th $m^{\prime} h$.

On détermine les coefficients $a_{0}^{\prime}$ et $a^{\prime}$. pour satisfaire à la condition (3), d'où :

$$
\begin{gathered}
\frac{a_{0}^{\prime}}{e^{2}} \frac{\operatorname{ch~} m_{0}^{\prime} y_{0}}{\operatorname{sh~} m_{0}^{\prime} h}-\sum_{1}^{\infty} \frac{2 i \alpha_{r}^{\prime}}{e^{2} \operatorname{sh} m_{r}^{\prime} h} \operatorname{ch} m_{r}^{\prime}, y_{0}=\mathrm{F}_{2}\left(y_{0}\right) \\
\frac{a_{0}^{\prime}}{e^{2} \operatorname{sh~} m_{0}^{\prime} h}=\frac{2 m_{0}^{\prime} \int_{0}^{h} F_{2}^{\infty}\left(y_{0}\right) \operatorname{ch~} m_{0}^{\prime} y_{0} d y_{0}}{m_{0}^{\prime} h+\operatorname{sh} m_{0}^{\prime} h \operatorname{ch~} m_{0}^{\prime} h} \frac{2 a_{r}^{\prime}}{e^{2} \operatorname{sh} m_{r}^{\prime} h}=\frac{2 i m_{r}^{\prime} \int_{0}^{h} F_{2}\left(y_{0}\right) \operatorname{ch~} m_{r}^{\prime}, y_{0} d y_{0}}{m_{r}^{\prime} h+\operatorname{sh} m_{r}^{\prime} h \operatorname{ch} m_{r}^{\prime} h}
\end{gathered}
$$

$3^{\circ}$ Recherche de LA Fonction $\mathrm{J}^{\prime \prime \prime}{ }_{2}\left(x_{0}, y_{0}, t\right)$ :

On aura de même :

$$
\begin{aligned}
& \mathrm{J}^{\prime \prime \prime}{ }_{3}\left(x_{0}, y_{0}, t\right)=-\frac{b_{0}^{\prime}}{m_{0}^{\prime} e^{2}} \frac{\operatorname{ch} m_{0}^{\prime} y_{0}}{\operatorname{sh~} m_{0}^{\prime} h} \sin \left(2 k t-m_{0}^{\prime} x_{0}\right)+\sum_{1}^{\infty} \frac{2 b_{r}^{\prime}}{m_{r}^{\prime} e^{2}} e^{-i m_{r}^{\prime} x_{0}} \frac{\operatorname{ch} m_{r}^{\prime} y_{0}}{\operatorname{sh} m_{r}^{\prime} h} \cos 2 k t \\
& \frac{b_{0}^{\prime}}{e^{2} \operatorname{sh} m_{0}^{\prime} h} \operatorname{ch~} m_{0}^{\prime} y_{0}-\sum_{1}^{z} \frac{2 i b_{r}^{\prime}}{e^{2} \operatorname{sh} m_{r}^{\prime} h} \operatorname{ch~} m_{r}^{\prime} I_{0}=\mathrm{F}_{3}\left(y_{0}\right) \\
& \frac{b_{0}^{\prime}}{e^{2} \operatorname{sh} m_{0}^{\prime} h}=\frac{2 m_{0}^{\prime} \int_{0}^{h} F_{3}\left(y_{0}\right) \operatorname{ch~} m_{0}^{\prime} y_{0} d y_{0}}{m_{0}^{\prime} h+\operatorname{sh} m_{0}^{\prime} h h \operatorname{ch~} m_{0}^{\prime} h} \frac{2 b_{r}^{\prime}}{e^{2} \operatorname{sh} m_{r}^{\prime} h}=\frac{2 i m_{r}^{\prime} \int_{0}^{h} F_{3}\left(y_{0}\right) \operatorname{ch~} m_{r}^{\prime}, y_{0} d y_{0}}{m_{r}^{\prime} h+\operatorname{sh} m_{r}^{\prime} h \operatorname{ch~} m_{r}^{\prime} h}
\end{aligned}
$$

Le calcul des coefficients $a_{0}^{\prime}, b_{0}^{\prime}, a_{r}^{\prime}, b_{r}^{\prime}, c_{r}^{\prime}$ se ramène au calcul d'intégrales de la forme : 


$$
\begin{gathered}
\mathrm{I}=\int_{i}^{\prime \prime} \operatorname{ch} \alpha y_{0} \operatorname{ch} \beta y_{0} d y_{0}=\frac{\operatorname{sh}(\alpha+\beta) h}{2(\alpha+\beta)}+\frac{\operatorname{sh}(\alpha-\beta) h}{2(\alpha-\beta)} \\
\mathrm{J}=\int_{0}^{\prime \prime} \operatorname{sh} \alpha y_{0} \operatorname{ch} \beta y_{0} d y_{0}=\frac{\operatorname{ch}(\alpha+\beta) h}{2(\alpha+\beta)}+\frac{\operatorname{ch}(\alpha-\beta) h}{2(\alpha-\beta)}-\frac{\alpha}{\alpha^{2}-\beta^{2}}
\end{gathered}
$$

dans lesquelles on remplacera $\alpha$ par $m_{0}, 2 m_{0}, m_{p}, m_{0} \pm m_{p}, m_{p} \pm m_{q}$ et $\beta$ par $i \mu^{\prime}, m_{0}^{\prime}, m^{\prime}{ }_{r}$.

On peut simplifier l'expression des coefficients en utilisant les formules d'addition pour les fonctions hyperboliques et en remarquant que :

$$
\begin{gathered}
\cos \mu_{n} h=(-1)^{r+1} \quad \sin \mu_{p} h=0 \\
\text { th } m_{0} h=\frac{k^{2}}{m_{0} g} \quad \text { th } m_{p} h=\frac{k^{2}}{m_{p} g} \quad \text { th } m_{0}^{\prime} h=\frac{4 k^{2}}{m_{0}^{\prime} g}
\end{gathered}
$$

Les calculs ne présentent aucune difficulté. Ils sont d'ailleurs grandement facilités par les symétries existant entre certains termes qui se déduisent les uns des autres par changement de :

$$
m_{p} \text { en }-m_{p} \text {, } m_{0} \text { en } m_{p} \text {, etc... }
$$

On obtient ainsi les résultats figurant à la planche I. On a pris dans ces formules :

$$
f\left(y_{0}\right)=1+\frac{\Delta e}{h e} y_{0} \text { d'où } f^{\prime}\left(y_{0}\right)=\frac{\Delta e}{h e}
$$

Le cas đu batteur piston s'obtient en prenant $\Delta e=0$ aussi bien dans les formules donnant $a_{0}^{\prime}, b_{0}^{\prime}, c^{\prime}{ }_{r}$ que dans les formules donnant $A_{0}, A_{p}$.

Les formules domnant les coefficients $a_{r}^{\prime}$ et $b_{r}^{\prime}$ se déduisent des formules donnant $a_{0}^{\prime}$ et $b_{0}^{\prime}$ par simple changement de $m_{0}^{\prime}$ en $m_{r}^{\prime}=-i \kappa_{r}^{\prime}$ et multiplication par $i / 2$.

Il est donc inutile de les écrire.

Dans ces formules, on a posé :

$$
\mathrm{A}_{0}=\frac{a_{0}}{e} \quad \mathrm{~A}_{j}=\frac{a_{p}}{e} \quad \mathrm{~L}_{0}=m_{0} h \quad \mathrm{I}_{\prime \prime}^{\prime}=m_{0}^{\prime} h_{1} \quad \mathrm{M}_{,}=\omega_{p} h \quad \mathrm{~L}_{p}=\lambda_{\nu p} h \quad \mathrm{R}=\frac{k^{2} h}{g}
$$

Ce sont des grandeurs sans dimension et l'on reconnaît aisément que $h c^{\prime}{ }_{2} / e^{2}$, $h a_{0}^{\prime} / e^{2}$ et $h b_{0}^{\prime} / e^{2}$ sont elles-mêmes sans dimensions.

On remarque que les séries numériques donnant les divers coefficients sont rapidement convergentes. Leur terme général est de l'ordre de :

$$
\begin{gathered}
\frac{1}{p^{3}} \text { ou } \frac{1}{p^{4}} \\
\mathrm{~L}_{p}=\lambda_{p} h \sim p \pi \quad \frac{a_{p}}{\sin \lambda_{p} h}=\mathrm{O}\left(\frac{1}{p^{2}}\right) \text { ou } \mathrm{O}\left(\frac{1}{p^{3}}\right)
\end{gathered}
$$

Il est done possible de calculer ces coefficients avec une bonne précision tout en se contentant d'un nombre limité de termes. On peut d'ailleurs trouver facilement une valeur approchée par excès du reste de chaque série, ce qui permet de chiffrer l'erreur commise.

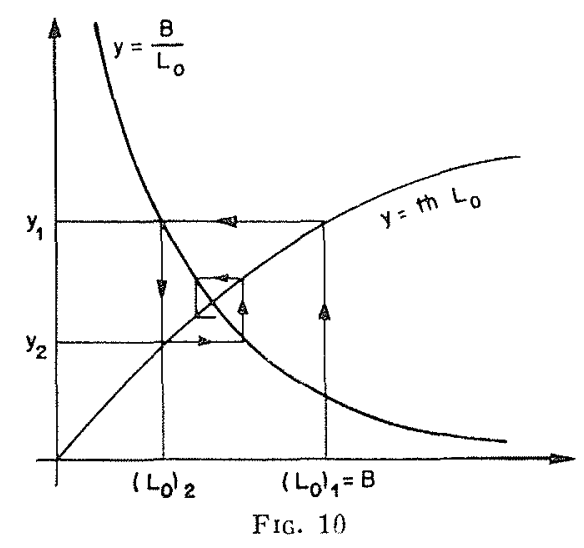


Pour effectuer le calcul proprement dit, il faut d'abord déterminer la valeur des racines :

$$
\begin{aligned}
\mathrm{L}_{0}=m_{0} h & \mathrm{~L}_{p}=\lambda_{p} h \\
\mathrm{~L}_{0}^{\prime}=m_{0}^{\prime} h & \mathrm{~L}^{\prime}{ }_{r}=\lambda_{,}^{\prime} h
\end{aligned}
$$

On procède par itération.

On commence les itérations avec la valeur approchée :

On calcule :

$$
\left(\mathrm{L}_{0}\right)_{1}=\left(m_{0} h\right)_{1}=\frac{k^{2} h}{g}=\mathrm{B}
$$

$$
y_{1}=\operatorname{th}\left(\mathrm{L}_{0}\right)_{1}
$$

On en déduit la nouvelle valeur:

On calcule :

$$
\left(\mathrm{L}_{0}\right)_{2}=\frac{\mathrm{B}}{y_{1}}
$$

$$
y_{2}=\operatorname{th}\left(\mathrm{L}_{0}\right)_{2}
$$
courbes :

La figure montre clairement que les itérations convergent vers le point d'intersection des

$$
y=\operatorname{th~}_{0} \quad y=\frac{\mathrm{B}}{\mathrm{L}_{0}}
$$

c'està-dire vers la racine cherchée. On arrête les itérations quand la différence entre deux valeurs successives de $L_{0}$ devient inférieure à la précision désirée.

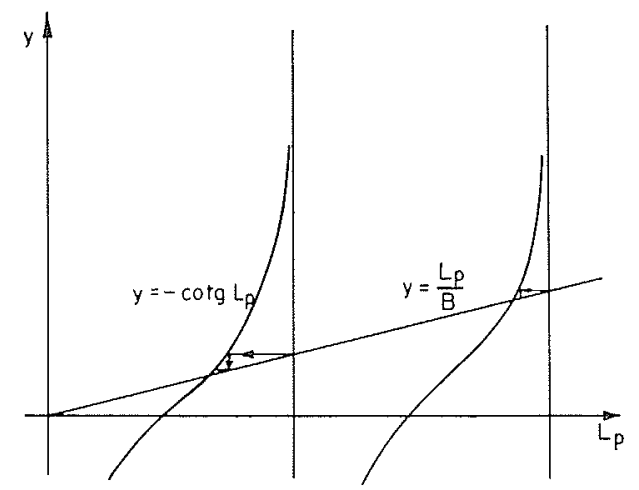

FIG. 11 $\left(\mathrm{L}_{p}\right)_{1}=p \pi$.

De même, pour le calcul de $\mathrm{L}_{p}$, on commence les itérations avec la valeur approchée

On calcule :

On en déduit :

$$
y_{1}=\frac{g}{k^{2} h}(\lambda h)_{1}=\frac{\left(\mathrm{L}_{p}\right)_{1}}{\mathrm{~B}}
$$

$$
\left(\mathrm{L}_{p}\right)_{2}=-\operatorname{arctg} \frac{1}{y_{1}}
$$

Les itérations convergent encore vers le point d'intersection des courbes :

$$
y=-\operatorname{cotg} \mathrm{L}_{p} \text { et } y=\mathrm{L}_{p} / \mathrm{R}
$$

L'application des formules donne alors :

$$
\frac{h a_{0}^{\prime}}{e^{2}}, \quad \frac{h b_{0}^{\prime}}{e^{2}}, \quad \frac{h c_{r}^{\prime}{ }_{r}}{e^{2}}, \quad \frac{h a_{r}^{\prime}}{e^{2}}, \quad \frac{h b_{r}^{\prime}}{e^{2}}
$$

en fonction de $L_{0}, L_{0}^{\prime}, L_{p}$ et $B$. 
Pour construire les abaques, point par point, on prend pour variable indépendante la profondeur relative $h / \mathrm{L}$ (variable sans dimensions), L étant la longueur d'onde de la houle fondamentale.

On a d'ailleurs :

$$
\begin{gathered}
\mathrm{L}_{0}=m_{0} h=\frac{2 \pi h}{\mathrm{~L}} \\
\mathrm{~B}=\frac{k^{2} h}{g}=\mathrm{L}_{0} \text { th } \mathrm{L}_{0}=f\left(\frac{h}{\mathrm{~L}}\right)
\end{gathered}
$$

De même, $\mathrm{L}_{0}^{\prime}, \mathrm{L}_{j}$, ne dépendent que de $\mathrm{B}$ et donc de $h / \mathrm{L}$.

Il en résulte que $h a_{0}^{\prime} / e^{2}, h b_{0}^{\prime} / e^{2} \ldots$ ne dépendent que de $h / \mathrm{L}$ et de $\Delta e / e$.

Pour traiter le cas du volet balancoire d'équation ef $\left(y_{0}\right)=e+(\Delta e / h) y_{0}$ avec un rapport $\Delta e / c$ quelconque, il est possible, dans l'approximation du 1 ordre, de décomposer chaque coefficient $a_{0}, a_{p}$ en deux parties, la première correspondant au batteur piston $(\Delta e=0)$, et la denxième au volet simple avec centre de rotation sur le fond $(e=0)$. Ainsi, pour chaque coefficient, il suffit de tracer deux abaques seulement.

Au second ordre, par contre, les formules donnant $h a_{0}^{\prime} / e^{2}$, etc., n'étant pas linéaires par rapport à $A_{0}, A_{p}$, il n'est pas possible de procéder de la sorte et l'on doit tracer pour chaque coefficient $h a_{0}^{\prime} / e^{2}, h b_{0}^{\prime} / e^{2}$, etc., un réseau d'abaque dépendant du paramètre $\Delta e / e$.

En fait, nous n'avons calculé que les abaques correspondant à la valeur $\Delta e / e=0$ (batteur piston). Ceux-ci donnent, en fonction de la profondeur relative $h / L$, l'amplitude de l'harmonique, soit :

$$
\frac{h \mathrm{~A}_{0}^{\prime}}{e^{2}}=\frac{h \sqrt{a^{\prime 2} 0+b^{\prime 2}}}{e^{2}}
$$

et sa différence de phase a avec le terme du 2 ordre associé à la houle fondamentale (*), cette différence de phase étant calculée au droit du batteur $\left(x_{0}=0\right)$.

$$
\alpha=\operatorname{arctg} \frac{b_{0}^{\prime}}{a_{0}^{\prime}}+\pi
$$

On a ainsi le diagramme:

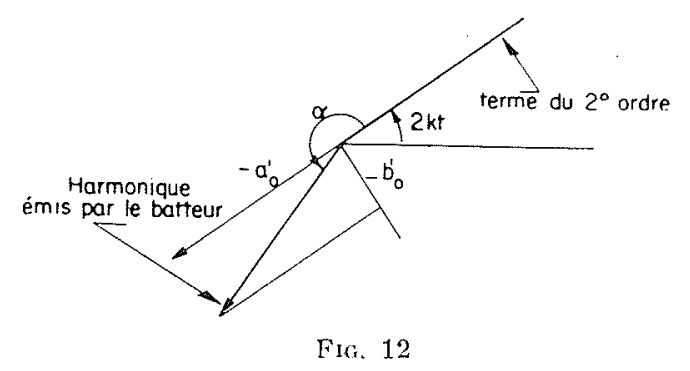

Il est facile d'interpréter la forme de ces abaques. Si la profondeur relative est faible, on est lans le cas des ondes longues en eau peu profonde (ch $m_{0} h \# 1$ ). La houle épouse alors assez bien le mouvement du batteur piston et loscillation locale garde une très petite amplitude. L'harmonique émis par le hatteur sert principalement à compenser le terme du 2" ordre de la houle. Ceci explique qu'il soit pratiquement en opposition de phase avec ce demier. Si $h / L$ croît, le mouvement de la houle épouse moins bien celui du batteur. L'oscillation locale qui fait la compensation devient plus importante et concourt, en même temps que le terme de M. Miche, à la formation de I'harmonique. La phase de ce dernier devient donc quelconque par rapport au premier.

(") Ce terme a pour abscisse:

$$
\frac{-m_{0} a_{0}^{2}}{4 e^{2} \operatorname{sh}^{2} m_{0} h}\left[1-\frac{3 \operatorname{ch} 2 m_{0} y_{0}}{2 \operatorname{sh}^{2} m_{0} h}\right] \sin \left(2 k t-2 m_{0} x_{0}\right)
$$


Lorsque $h / \mathrm{L}$ croît au-dessus de 0,5 , la profondeur peut être considérée pratiquement comme infinie. Une augmentation de $h / \mathrm{L}$ n'a donc pas d'effet sensible sur le mouvement. Ceci explique la présence d'une asymptote pour la phase et pour l'amplitude de l'harmonique (*).

Lorsque $h / L$ est faible, l'amplitude du terme de $M$. Miche est importante puisqu'elle croît en raison inverse de la profondeur. L'amplitude de l'harmonique qui est engendrée par le terme devient donc elle aussi importante.

Au-dessous d'une valeur minimale de $h / \mathrm{L}$, la méthode des petits paramètres qui nous a servi à expliciter la solution cesse d'être valable, car les termes du $2^{\circ}$ ordre croissent au-delà de toute limite et la série qui a servi à représenter le mouvement du fluide :

$$
\left(x=x_{01}+e \mathrm{X}_{1}+e^{2} \mathrm{X}_{2}+\ldots\right)
$$

n’est probablement plus convergente. La partie gauche des abaques devra donc être utilisée avec précaution.

Des expériences sont en cours au Laboratoire de Mécanique des Fluides de l'Institut Polytechnique de Grenoble. Elles auront pour but de vérifier la présente théorie et de déterminer ses limites de validité.

Lorsque le mouvement du batteur n'est pas rigoureusement sinusoïdal, l'harmonique calculé par la présente théorie se compose avec l'harmonique calculé par M. Apté. Ces deux termes étant du 2 ordre, on a vu qu'ils s'ajoutent vectoriellement comme si le phénomène était linéaire, puisque leurs interactions sont d'ordre supérieur au second.

Les expériences demandent une mesure trés précise des phases et des amplitudes, mesure d'autant plus délicate que les dernicres sont de lordre de quelques millimètres.

En effet, pour une houle fondamentale ayant une longueur d'onde de 1 mètre, une cambrure de $5 \%$, et pour une profondeur d'eau de 30 centimotres, labaque donne une amplitude de $1,9 \mathrm{~mm}$ seulement $\left(2 \mathrm{~A}_{0}^{\prime}\right)$.

Les mesures sont effectuées avec l'appareil S.M. mis au point au Laboratoire de Mécanique des Fluides par M. le professeur Santon et par M. Marcou. L'extrême sensibilité de cet appareil permet de mettre en évidence les phènomènes du second ordre.

\section{CHAPITRE V}

\section{VALIDITE DE LA SOLUTION FORMELLE}

La présente théorie est basée sur la validité de la représentation des petits mouvements d'un fluide pesant à surface libre, par une série de la forme:

$$
\begin{gathered}
x=x_{0}+e \mathrm{X}_{1}\left(x_{0}, y_{0}, t\right)+e^{2} \mathrm{X}_{2}\left(x_{0}, y_{0}, t\right)+\ldots \\
y=y_{0}+e Y_{1}\left(x_{0}, y_{0}, t\right)+e^{2} \mathbf{Y}_{2}\left(x_{0}, y_{0}, t\right)+\ldots
\end{gathered}
$$

Rappelons que cette représentation est une application de la méthode des petits paramètres de Poincaré et qu'elle a été utilisée par M. Miche et à sa suite par MM. Biesel et Daubert. A notre connaissance, la convergence de ces séries n'a jamais été démontrée. On peut simplement remarquer que, dans la pratique, l'addition des termes du second ordre améliore nettement la description du mouvement. représentation.

La validité de la théorie du batteur dépendra donc essentiellement de la validité de cette

Nous avons admis ce fait, au début de notre travail, et nous avons supposé, en plus, qu'il

(") Pour déterminer le point à l'infini, il faudrait recommencer les calculs en utilisant les expressions de $X_{1}$ et $Y_{1}$ valables en profondeur infinie (cf. Havelock). Nous n'avons pas fait ces calculs étant donné leur faible intérêt pratique 
était possible de limiter la série à ses termes du $1^{\text {er }}$ et du $2^{\circ}$ ordres, pour des valeurs de $e$ « suffisamment petites », mais dont la grandeur n'est pas susceplible d'être évaluće avec précision.

Remarquons, en outre, que nous nous sommes limités à l'étude des monvements roisins du mouvement irrotationnel (en raison des hypothèses faites sur le comrant de masse). Ces mouvements correspondent, sans doute, à ceux qui sont observés en pratique dans un canal à houle. Un rotationnel arbitraire du $1^{\text {er }}$ ordre aurait fait apparaître des termes séculaires qui augmentent indéfiniment avec le temps. Dans ces conditions, on aurait pu craindre que le rayon de convergence soit fonction du temps et que les séries ci-dessus ne convergent que pour des intervalles de temps trop petits pour qu'on puisse les utiliser. Cette objection est d'ailleurs classique et a été formulèe par Poincaré.

La validité de la représentation du mouvement du fluide ayant été admise, il convient maintenant de montrer la légitimité des opérations formelles effectuées. Celles-ci nous ont conduit à exprimer la solution sous la forme d'une somme de séries infinies. Ces dernières ne définissent, en réalité, le mouvement, qu'à la condition d'être uniformément convergentes dans la masse du fluide, ainsi que leurs deux premières dérivées par rapport aux coordonnées $x$, $y$ et $t$. Lorsque cette condition est réalisée, les séries représentent en effet des fonctions définies et continues dans tout le domaine ainsi que leurs deux premières dérivées. Ces fonctions vérifient d'ailleurs, par construction, les écuations du mouvement.

De la même manière, pour que les conditions aux limites soient effectivement satisfaites, il faut que les séries définissant $Y_{2}$ sur le fond du canal, $\partial 2 X_{0} / \partial t^{2}$ et $\partial Y_{2} / \partial t$ sur la surface libre, $\mathrm{X}_{2}$ et $\mathrm{Y}_{2}$ à l'infini, $\mathrm{X}_{2}$ sur le batteur soient miformément convergentes sur ces frontières.

Or la solution se compose de trois séries distinctes :

$$
\begin{aligned}
& \mathrm{X}_{2}=\frac{\partial \mathrm{I}_{2}}{\partial x_{0}}+\frac{\partial K_{2}}{\partial x_{0}}+\frac{\partial \mathrm{J}_{2}}{\partial x_{0}} \\
& Y_{2}=\frac{\partial \mathrm{I}_{2}}{\partial y_{0}}+\frac{\partial K_{2}}{\partial y_{0}}+\frac{\partial \mathbf{J}_{2}}{\partial y_{0}}
\end{aligned}
$$

Il est facile de montrer que les deux prenières sont uniformément convergentes ainsi que leurs dérivées dans la masse, sur le fond, à l'infini et sur la surface libre.

En effet, la solution au $1^{\text {er }}$ ordre, $X_{1}$ et $Y_{1}$, est donnée sous la forme de séries infinies absolument et uniformément convergentes ainsi que leurs dérivées de tous ordres, et cela, dans tonte la masse du fluide, ainsi que sur les frontières (batteur excepté).

Il est donc légitime d'effectuer les produits terme à terme des séries dérivées comme on l'a fait notamment pour calculer les jacobiens figurant aux seconds membres des équations, ou pour expliciter la condition limite en surface. On obtient ainsi des séries qui sont absolument et uniformément convergentes dans tout le domaine sauf peut-être sur le batteur. A leur tour, les séries :

$$
\frac{\partial \mathrm{L}_{2}}{\partial x_{0}}, \quad \frac{\partial \mathrm{L}_{2}}{\partial y_{0}}, \quad \frac{\partial \mathrm{K}_{2}}{\partial x_{0}}, \quad \frac{\partial \mathrm{K}_{2}}{\partial y_{0}}
$$

sont obtenues en intégrant, terme à terme, les séries uniformément convergentes qui constituent les seconds membres des équations du mourement ou de la condition de surface. Elles sont donc absolument et uniformement convergentes dans toute la masse du fluide sauf peut-être sur le batteur. On peut d'ailleurs le vérifier directement sur l'expression de $\mathrm{X}_{2}$ et $\mathrm{Y}_{2}$ lorsque l'on a remplacé les $\mathrm{M}_{p}$ et $\mathrm{M}_{n}$ par leurs valeurs et regroupé les termes. Ceux-ci contiennent en facteur les termes exponentiels $e^{-\lambda_{p} x_{0}}, e^{-\lambda_{q} x_{0}}$, qui assurent la convergence des fonctions et de leurs dérivées de tous ordres quel que soit $x_{0}$ positif.

Par contre, sur le batteur, $x_{0}=0$, les exponentielles se réduisent à l'unité et l'on doit examiner la convergence de plus près.

On fera ci-dessous l'étude de la série $\xi\left(0, y_{0}, t\right)$ à laquelle se réduit l'expression $\left(\partial L_{2} / \partial x_{0}\right)+\left(\partial K_{2} / \partial x_{0}\right)$ sur le volet. On verra qu'elle y est uniformément convergente sauf aux extrémités et au point milieu $\left(x_{0}=0, y_{0}=h / 2\right)$, où la fonction représentée par la série possède des singularités logarithmiques, de partie principale $\log z_{0}, \log \left(z_{0}-i h\right), \log \left(z_{0}-i h / 2\right)$. série :

Il reste à étudier la convergence dans la masse et sur les frontières de la troisième

$$
\mathrm{X}_{2}^{\prime \prime}=\frac{\partial \mathrm{J}_{2}}{\partial x_{0}} \quad \mathbf{Y}_{2}^{\prime \prime}=\frac{\partial \mathrm{J}_{2}}{\partial y_{0}}
$$


Une étude analogue à celle qui a déjà été faite au $1^{\mathrm{er}}$ ordre montrerait que leur convergence dépend de la nature des singularités de la fonction qu'elles représentent sur le batteur, soit :

$$
\left[\mathrm{X}_{2}-\frac{\partial \mathrm{L}_{2}}{\partial x_{0}}-\frac{\partial \mathrm{K}_{2}}{\partial x_{0}}\right]_{r_{0}=0}=\mathrm{X}_{2}-\xi\left(0, y_{0}, t\right)
$$

On connait déjà les singularités de $\xi\left(0, y_{0}, t\right)$ et l'on devra rechercher également celles de la solution au $2^{e}$ ordre $\mathrm{X}_{2}$ et $\mathrm{Y}_{2}$. On verra que celles-ci sont situées aux extrémités du batteur et ont pour partie principale $z_{0}\left(\log z_{0}\right)^{2}$, le point milieu étant, d'ailleurs, un point ordinaire.

On en conclut que $\partial \mathbf{J}_{2} / \partial x_{0}$ étant égale sur le batteur à une tonction de carré intégrable, y est uniformément convergente dans tout intervalle ne contenant pas les singularités; c'esta-dire, dans les intervalles ouverts $(0, h / 2)$ et $(h / 2, h)$.

En outre, dans la masse, sur le fond, a l'infini, et sur la surface libre, on montrerait, comme pour l'approximation du 1 er ordre, que les séries $\left(\partial \mathrm{J}_{2} / \partial x_{0}\right),\left(\partial \mathrm{I}_{2} / \partial y_{0}\right)$ ainsi que leurs dérivées de tous ordres sont absolument et uniformément convergentes. On peut remarquer que la solution particulière :

$$
\mathrm{X}_{2}^{\prime}=\frac{\partial \mathrm{L}_{2}}{\partial x_{0}}+\frac{\partial \mathrm{K}_{2}}{\partial x_{0}} \quad \mathrm{Y}_{2}^{\prime}=\frac{\partial \mathrm{L}_{2}}{\partial y_{0}}+\frac{\partial \mathrm{K}_{2}}{\partial y_{0}}
$$

satisfait aux conditions de fond, à l'infini, et à la surface libre, mais qu'elle prend sur le batteur des valcurs très particulières. On aurait pu obtenir une infinité d'autres solutions particulières, satisfaisant aux mêmes conditions de fond, de surface, et à l'infini, mais prenant chacune sur le batteur des valeurs diférentes et y possédant éventuellement des singularités arbitraires. Ces diverses solutions ne différeraient les unes des autres que par le gradient d'une fonction harmonique, vérifiant les trois premières conditions limites. Ainsi les singularités de :

$$
\left(\partial I_{2} / \partial x_{0}\right)+\left(\partial K_{2} / \partial x_{0}\right)
$$

ne correspondent pas nécessairement à la nature du problème et dépendent en partie du choix de la solution particulière. Ces singularités artificiclles se retrouvent d'ailleurs avec le signe contraire dans la série $\partial \mathrm{J}_{2} / \partial x_{0}$, puisque celle-ci est déterminée de telle manière que pour $x_{0}=0$

$$
\frac{\partial J_{2}}{\partial x_{0}}=X_{2}-\frac{\partial I_{2}}{\partial x_{0}}-\frac{\partial K_{2}}{\partial x_{0}}
$$

Elles se détruisent done mutuellement dans le calcul de la solution complète $\mathrm{X}_{2}$ et $\mathrm{Y}_{\underline{2}}$. Par contre, les singularités de cette dernière sont parfatement déterminées et irréductibles, puisqu'elles sont dans la nature des choses. Elles sont d'ailleurs plus faibles que les premières puisqu'elles sont en $z_{0}\left(\log z_{0}\right)^{2}$ et $\left(z_{0}-\ldots i h\right)\left[\log \left(z_{0} \ldots i h\right)\right]^{2}$.

Ainsi, on reconnaît que les déplacements $X_{.,}, Y_{2}$ sont partout finis et continus, milieu et extrémités du batteur compris, bien que les séries:

$$
\frac{\partial \mathrm{L}_{2}}{\partial x_{0}}+\frac{\partial \mathrm{K}_{2}}{\partial x_{0}} \text { et } \frac{\partial \mathrm{J}_{2}}{\partial x_{0}}
$$

y prennent des valeurs infinies (mais de signes contraires).

De plus, la partie principale de $\mathrm{X}_{2}, \mathrm{Y}_{2}$ étant $z_{0}\left(\log z_{0}\right)^{2}$, les séries dérivées $\left(\partial \mathrm{X}_{2} / \partial y_{0}\right)$, $\left(\partial Y_{2} / \partial y_{0}\right)$ ont des singularités logarithmiques et les séries qui les représentent sont uniformément convergentes.

Les séries représentant $\mathrm{X}_{2}$ et $\mathrm{Y}_{2}$, obtenues par intégration des séries précédentes, sont done absolument convergentes, bien que les séries $\left(\partial \mathbf{L}_{2} / \partial x_{0}\right)+\left(\partial K_{2} / \partial x_{0}\right)$ d'une part et $\partial \mathrm{J}_{2} / \partial x_{0}$ d'autre part soient simpJement uniformément convergentes. Leur somme, après réarrangement des termes, possède un terme général d'ordre $1 / p^{2}$. On pourrait profiter de ces remarques pour effectuer plus adroitement le calcul, en évitant de décomposer $X_{2}$ et $Y_{2}$ comme on l'a fait ci-dessus, en composantes prenant en certains points des valeurs infinies et de signe contraire. On choisirait pour cela, pour solution particulière :

$$
\mathrm{X}_{2}^{\prime}=\frac{\partial \mathrm{L}_{2}}{\partial x_{0}}+\frac{\partial \mathrm{K}_{2}}{\partial x_{0}}+\frac{\partial \mathrm{I}_{2}}{\partial x_{0}} \quad \mathbf{Y}_{2}^{\prime}=\frac{\partial \mathrm{L}_{2}}{\partial y_{0}}+\frac{\partial \mathrm{K}_{2}}{\partial y_{0}}+\frac{\partial \mathrm{I}_{2}}{\partial y_{0}}
$$


ò̀ $\mathrm{I}_{2}$ est une fonction harmonique satisfaisant aux trois premières conditions aux limites et possédant sur le batteur des singularités égales aux singularités de $\left(\partial \mathrm{L}_{2} / \partial x_{0}\right)+\left(\partial \mathrm{K}_{2} / \partial x_{0}\right)$ et de signes contraires. On obtiendrait ainsi poux $\left(\partial \mathbf{L}_{2} / \partial x_{0}\right)+\left(\partial K^{2} / \partial x_{0}\right)+\left(\partial \mathbf{I}_{2} / \partial x_{0}\right)$, d'une part, et pour $\partial J_{2} / \partial x_{0}$, d'autre part, des séries absolument convergentes, plus agréables à manier dans les applications numériques.

Mais le gain ne serait appréciable que pour le calcul de l'oscillation locale du 2" ordre, à laquelle nous ne nous intéressons pas puisqu'elle devient négligeable à très faible distance du batteur. Comme nous nous contentons, en fait, du calcul de l'amplitude et de la phase du terme progressif, nous avons préféré utiliser la solution particulière uniformément convergente.

On peut conclure cette discussion en remarquant que la solution formelle, donnée au chapitre précédent fournit bien la représentation du mouvement du fluide dans le canal, au 2 ordre d'approximation, les déplacements calculés étant partout finis et continus, tandis que leurs dérivées présentent des infinis logarithmiques aux extrémités du hatteur comme dans la solution au $1^{\mathrm{er}}$ ordre.

On procédera maintenant, dans les paragraphes suivants, à l'étude de la convergence des séries $\xi\left(0, y_{0}, t\right)$ et à la détermination des singularités des fonctions $\mathrm{X}_{2}$ et $\mathrm{Y}_{2}$.

Etude de LA Convergexce de da série $\zeta\left(0, y_{0}, t\right)$ :

Cette série se décompose en plusieurs séries que nous étudierons séparément.

a) Séries : $u_{p}=\frac{a_{0}}{4 c^{2} \operatorname{sh} m_{0} h} \sum_{1} \frac{a_{p}}{\operatorname{sh} m_{p} h}\left(\begin{array}{r}{\left[\left(m_{0}-m_{p}\right)-\bar{y}_{0 p}\left(m_{0}+m_{p}\right)\right] \operatorname{ch}\left(m_{0}+m_{p}\right) y_{0}} \\ -\left[\left(m_{0}+m_{p}\right)-p_{0 p}\left(m_{0}-m_{p}\right)\right] \operatorname{ch}\left(m_{0}-m_{p}\right) y_{0}\end{array}\right\}$

Il est facile de développer les deux expressions complexes conjuguées :

$$
\begin{aligned}
& \left(m_{0}+m_{p}\right)-\mu_{0 p}\left(m_{0}-m_{p}\right)=-2 \frac{k^{4}+g^{2} m_{p}{ }^{2}}{g^{2}\left(m_{0}-m_{p}\right)}=2 m_{p}\left[1+\frac{m_{0}}{m_{p}}+\frac{m_{0}{ }^{2}+k^{4}}{m_{p}{ }^{2}}+\cdots\right] \\
& \left(m_{0}-m_{p}\right)-\bar{\mu}_{0 p}\left(m_{0}+m_{p}\right)=-2 \frac{k^{4}+g^{2} m_{p}{ }^{2}}{g^{2}\left(m_{0}+m_{p}\right)}=-2 m_{p}\left[1-\frac{m_{0}}{m_{p}}+\frac{m_{0}{ }^{2}+k^{4}}{m_{p}{ }^{2}}+\cdots\right]
\end{aligned}
$$

On a donc :

$$
u_{p}=\frac{a_{0} \operatorname{ch} m_{0} y_{0}}{e \operatorname{sh} m_{0} h} \sum_{1}^{\infty}\left[-\frac{\lambda_{p} a_{p}}{e \sin \lambda_{p} h} \cos \lambda_{p} y_{0}+\mathrm{O}\left(1 / p^{2}\right)\right]
$$

qui peut s'écrire encore, compte tenu de l'expression développée de $a_{p} / \sin \lambda_{p} h$ (voir théorie au $1^{\text {er }}$ ordre) et de la valeur de $\lambda_{p}=(p \pi / h)+\mathrm{O}(1 / p)$.

$$
u_{p}=\frac{a_{0} \operatorname{ch} m_{0} y_{0}}{e \operatorname{sh} m_{0} h} \sum_{1}^{\infty}\left[\frac{2}{\pi h^{2}}\left((-1)^{p}\left[\frac{k^{*}}{g} f(h)-f^{\prime}(h)\right]+f^{\prime}(o) \frac{\cos p \alpha}{p}+\mathrm{O}\left(1 / p^{2}\right)\right]\right.
$$

où l'on a posé $\alpha=\pi y_{0} / h$.

La série $u_{p}$ converge ou diverge en même temps que les séries dont le terme général est de la forme $(-1)^{p} K_{1}(\cos p \alpha / p)$ ou $K_{2}(\cos p \alpha / p)$. En effet, elle n'en diffère que par une série absolument convergente.

En posant $\beta=\pi-\alpha$, la première série, dans laquelle les coefficients du cosinus ont des signes alternés, se ramène à une série à coefficients de même signe. Or, en vertu du théorème d'Abel, ces séries sont uniformément convergentes sur l'intervalle ouvert :

$$
\begin{aligned}
& 0<\alpha<2 \pi \text { soit } \quad 0<y_{0}<2 h \\
& 0<\beta<2 \pi \text { soit }-h<y_{0}<+h
\end{aligned}
$$

En résumé, si la fonction $f\left(y_{0}\right)$ ne satisfait pas aux conditions limites de fond et de surface, la série $u_{p}$ est uniformément convergente dans l'intervalle $(o, h)$ extrémités exclues. Dans le cas contraire, la série $u_{p}$ est absolument et uniformément convergente dans l'intervalle $(o, h)$ extrémités comprises. 
b) On développerait de la même façon les expressions :

$$
\begin{gathered}
m_{0}-m_{p}-\lambda_{0 p}\left(m_{0}+m_{p}\right)=\frac{10 m_{0}\left(k^{4}-m_{p}^{2} g^{2}\right)+2 m_{p}\left(k^{4}-m_{0}^{2} g^{2}\right)+2 m_{0} g^{2}\left(m_{p}{ }^{2}-m_{0}{ }^{2}\right)}{4 k^{4}-g^{2}\left(m_{0}-m_{p}\right)^{2}} \\
m_{0}-m_{p}+\lambda_{0 p}\left(m_{0}+m_{p}\right)=-\frac{10 m_{p}\left(k^{1}-m_{0}^{2} g^{2}\right)+2 m_{0}\left(k^{4}-m_{p}^{2} g^{2}\right)+2 m_{p} g^{2}\left(m_{0}{ }^{2}-m_{p}{ }^{2}\right)}{4 k^{4}-g^{2}\left(m_{0}-m_{p}\right)^{2}}
\end{gathered}
$$

et les quantités complexes conjuguées.

Les autres séries, représentant l'interaction de la houle fondamentale avec l'oscillation locale, peuvent donc se mettre sous la forme:

$$
\begin{gathered}
\left.-\sum_{1}^{\infty} \frac{i a_{0} a_{p}}{4 e^{2} \operatorname{sh~} m_{0} h \operatorname{sh} m_{p} h !+\left[\left(m_{0}+m_{p}\right)-\bar{\lambda}_{0 p}\left(m_{0}-m_{p}\right)\right] \operatorname{ch}\left(m_{0}-m_{p}\right) y_{0}}\right\} \\
=\frac{4 a_{0} m_{0}}{e \operatorname{th} m_{0} h} \sum_{1}^{\infty}\left[\frac{a_{p}}{e \sin \lambda_{p} h} \cos \lambda_{p} y_{0}+\mathrm{O}\left(1 / p^{2}\right)\right]
\end{gathered}
$$

qui est absolument convergente, même si $f\left(y_{0}\right)$ ne satisfait pas aux conditions aux limites, et :

$$
\begin{aligned}
& \sum_{1}^{\infty} \frac{a_{0} a_{n}}{4 e^{2} \operatorname{sh~} m_{0} h \operatorname{sh} m_{p} h}: \begin{array}{c}
{\left[\left(m_{0}-m_{n}\right)+\lambda_{0,}\left(m_{0}+m_{n}\right)\right] \operatorname{ch}\left(m_{0}+m_{n}\right) y_{0}} \\
{\left[\left(m_{0}+m_{p}\right)+\lambda_{n j}\left(m_{0}-m_{p}\right)\right] \operatorname{ch}\left(m_{0}-m_{p}\right) y_{0}}
\end{array} \\
& =-\frac{a_{0}}{e^{2} \operatorname{th} m_{0} h} \sum_{1}^{\infty}\left[\frac{\lambda_{p} a_{p}}{\sin \lambda_{p} h} \cos p \%+\mathrm{O}\left(1 / p^{2}\right)\right\rceil
\end{aligned}
$$

qui est uniformément convergente dans l'intervalle $0, h$ si $f\left(y_{0}\right)$ ne satisfait pas aux conditions aux limites, et absolument convergente dans l'intervalle fermé, daus le cas contraire.

$$
\text { c) Série } \sum_{1}^{\infty} \sum_{1}^{\infty} \frac{i a_{p} a_{q}\left(m_{p}+m_{q}\right)}{2 e^{2} \operatorname{sh} m_{p} h \operatorname{sh} m_{q} h}\left[\operatorname{ch}\left(m_{p}-m_{q}\right) y_{0}+u_{p q}^{\prime} \operatorname{ch}\left(m_{p}+m_{q}\right) y_{0}\right]
$$

En utilisant la relation $m_{p}=-i \lambda_{p}$, on peut écrire celte série sous forme réelle

$$
\sum_{1}^{\infty} \sum_{1}^{\infty} \mathrm{U}_{p q}=-\sum_{1}^{\infty} \sum_{1}^{\infty} \frac{a_{p} a_{q}\left(\lambda_{p}+\lambda_{q}\right)}{2 e^{2} \sin \lambda_{p} h \sin \lambda_{q} h}\left[\cos \left(\lambda_{p}-\lambda_{q}\right) y_{0}+\bar{\mu}_{p q}^{\prime} \cos \left(\lambda_{p}+\lambda_{q}\right) y_{0}\right]
$$

avec :

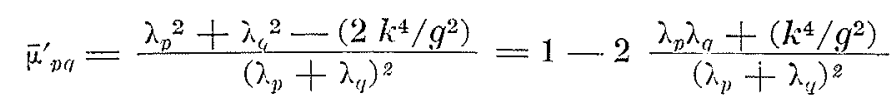

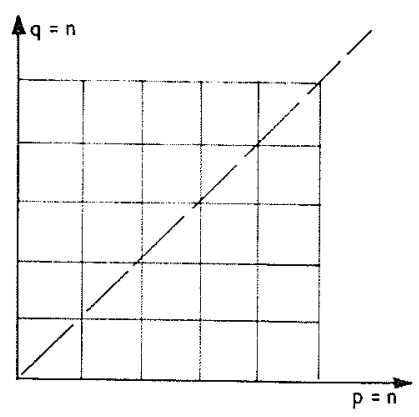

FIG. 13

On doit sommer dans tout le premier quadrant du plan $p$, $q$. On calculera la somme des termes contenus dans le carré de còté $n$ :

$$
\sum_{1}^{n} \sum_{1}^{n} \mathbf{U}_{m q}
$$

et l'on vérifiera que cette somme a une limite, lorsque :

$$
n \rightarrow \infty
$$


On a donc :

$$
\begin{aligned}
& \sum_{1}^{n} \sum_{1}^{n} \mathrm{U}_{p q}=-\sum_{1}^{n} \sum_{1}^{n} \frac{a_{p} a_{a}\left(\lambda_{p}+\lambda_{q}\right)}{e^{2} \sin \lambda_{p} h \sin \lambda_{q} h} \cos \lambda_{p} y_{0} \quad \cos \lambda_{q} y_{0} \\
& +\sum_{1}^{n} \sum_{1}^{n} \frac{a_{p} a_{q}}{e^{2} \sin \lambda_{p} h \sin \lambda_{q} h} \frac{\lambda_{p} \lambda_{q}+\left(K^{4} / g^{2}\right)}{\left(\lambda_{p}+\lambda_{q}\right)} \cos \left(\lambda_{p}+\lambda_{q}\right) y_{0}
\end{aligned}
$$

La première somme se décompose en deux produits de séries simples dont l'une est absolument convergente et l'autre uniformément convergente, soit :

$$
-\sum_{1}^{n} \frac{a_{p} \lambda_{p}}{e \sin \lambda_{p} h} \cos \lambda_{p} y_{0} \sum_{1}^{n} \frac{a_{u}}{e \sin \lambda_{q} h} \cos \lambda_{q} y_{0}
$$

et :

$$
-\sum_{1}^{n} \frac{a_{p}}{e \sin \lambda_{n} h} \cos \lambda_{p} y_{0} \sum_{1}^{n} \frac{a_{p} \lambda_{n}}{e \sin \lambda_{q} h} \cos \lambda_{, y} y_{0}
$$

La première somme de la série étudiée tend donc vers unc limite quel que soit $y_{0}$ dans l'intervalle ouvert $0, h$. Par contre, pour $y_{0}=0$ et $y_{0}=h$, elle devient infinie, à moins que $f\left(y_{0}\right)$ ne satisfasse aux conditions aux limites.

La deuxième somme s'étudic en même temps que la série

$$
\sum_{1}^{n} \sum_{1}^{n} \frac{a_{p} a_{0}}{e^{2} \sin \lambda_{n} h \sin \lambda_{q} h} \frac{\lambda_{n} \lambda_{r i}+\left(k^{4} / g^{2}\right)}{\left(\lambda_{p}+\lambda_{n}\right)} e^{i\left(\lambda_{p}+\lambda_{\eta}\right) y_{0}}
$$

qui peut se développer sous la forme :

$$
\frac{\pi}{h} \sum_{1}^{n}\left[(-1)^{p} \frac{\mathrm{R} \pi}{2 g}+f_{0}^{\prime}\right] \frac{e^{i p t}}{p^{2}} \sum_{1}^{n}\left[(-1) \frac{\mathrm{R} \pi}{2 g}+f_{0}^{\prime}\right] \frac{e^{i q(\sigma)}}{q^{2}} \frac{p q+\left(k^{!} h^{2}\right) /\left(g^{2} \pi^{2}\right)}{(p+q)}[1+\mathrm{O}(1 / p)+\mathrm{O}(1 / q)]
$$

Il suffit d'étudier le terme principal. D'autre part, puisqu'il est symétrique par rapport aux indices $p$ et $q$, on peut calculer cette expression en sommant dans la moitié du carré seulement, c'est-à dire en prenant les termes situés sur la diagonale principale d'une part et le double des termes situés en dessous de la diagonale:

$$
\sum_{1}^{n} \sum_{1}^{n 2} U_{p q}=\sum_{1}^{n} U_{p p}+2 \sum_{\geq 1}^{n} \sum_{1}^{p} U_{p r q}^{1}
$$

Il vient finalement :

$$
\sum_{1}^{n} \mathrm{U}_{n n}=\frac{\pi}{2 h} \sum_{1}^{n}\left[(-1) n \frac{\mathrm{R} \pi}{2 g}+f_{0}^{\prime}\right]^{2} \frac{p^{2}+\left(k h^{2}\right) /\left(g^{2} \pi^{2}\right)}{p^{5}} e^{2 i n}
$$

série absolument convergente,

et :

$$
\begin{aligned}
& 2 \sum_{1}^{n} \sum_{1}^{p} \mathrm{U}_{p q}=\frac{2 \pi}{h} \sum_{1}^{n}\left[(-1)^{p} \frac{\mathrm{R} \pi}{2 g}+f_{0}^{\prime}\right] \frac{e^{i p \alpha}}{p} \sum_{1}^{1}-1\left[(-1)^{-1} \frac{\mathrm{R} \pi}{2 g}+f_{0}^{\prime}\right] \frac{e^{i q \alpha}}{q(p+q)} \\
& \quad+\frac{2 k^{4} h}{g^{2} \pi} \sum_{1}^{n}\left[(-1)^{p} \frac{\mathrm{R} \pi}{2 g}+f_{0}^{\prime}\right] \frac{e^{i p \alpha}}{p^{2}} \sum_{1}^{p-1}\left[(-1)^{n} \frac{\mathrm{R} \pi}{2 g}+f_{0}^{\prime}\right] \frac{e^{i q \alpha}}{q^{2}(p+q)}
\end{aligned}
$$

La somme :

$$
\sum_{1}^{1,-1}\left[(-1)^{q} \frac{\mathrm{R} \pi}{2 g}+f_{0}^{\prime}\right] \frac{e^{i q \alpha}}{q(p+q)}
$$

peut se décomposer en deux, car :

$$
\frac{1}{q(p+q)}=\frac{1}{p q}-\frac{1}{p(p+q)}
$$


La première série se décompose donc à son tour en :

et :

$$
\frac{2 \pi}{h} \sum_{1}^{n}\left[(-1) p \frac{\mathrm{R} \pi}{2 g}+f_{0}^{\prime}\right] \frac{e^{i p \alpha}}{p^{2}} \sum_{1}^{n-1}\left[(-1) \frac{\mathrm{R} \pi}{2 g}+f_{0}^{\prime}\right] \frac{e^{i q_{n}}}{q}
$$

$$
\frac{2 \pi}{h} \sum_{1}^{n}\left[(-1)^{p} \frac{\mathrm{R} \pi}{2 g}+f_{0}^{\prime}\right] \frac{e^{i p \alpha}}{p^{2}} \sum_{1}^{\prime \prime}{ }^{1}\left[(-1)^{a} \frac{\mathrm{R} \pi}{2 g}+f_{0}^{\prime}\right] \frac{e^{i q \alpha}}{p+q}
$$

La somme :

$$
\mathrm{S}_{p}=\sum_{1}^{\prime \prime-1}\left[(-1)^{q} \frac{\mathrm{R} \pi}{2 g}+f_{0}^{\prime}\right] \frac{e^{i q q}}{q}
$$

est la somme des $p--1$, premiers termes d'une séric uniformément convergente dans l'intervalle ouvert $0, h$. Elle est donc bornée. Soit $\mathrm{S}$ sa somme.

La série :

$$
\left.\frac{2 \pi}{h} \sum_{1}^{n} \Gamma^{-}(-1)^{\prime} \frac{\mathrm{R} \pi}{2 g}+f_{0}^{\prime}\right] \cdot \frac{e^{i p \alpha}}{p^{2}} \mathrm{~S}_{n}
$$

est majorée par la sćrie absolument convergente :

$$
\frac{2 \pi}{h} \sum_{1}^{n}|| \frac{\mathrm{R} \pi}{2 g}\left|+f_{0}^{\prime}\right| \frac{\mathrm{S}}{p^{2}}
$$

En faisant le changement de variable $p+q=r$ dans la somme :

$$
\sum_{\eta=1}^{q=n-1}\left[(-1)^{q} \frac{\mathrm{R} \pi}{2 g}+f_{0}^{\prime}\right] \frac{e^{i q \alpha}}{p+q}=e^{-i / n \alpha} \sum_{r=p+1}^{r=2 p-1}\left[(-1)^{r-n} \frac{\mathrm{R} \pi}{2 g}+f_{0}^{\prime}\right] \frac{e^{i r \alpha}}{r}
$$

On voit que cette somme tend vers zéro dans l'intervalle ouvert $o, h$.

La série :

$$
\frac{2 \pi}{h} \sum_{1}^{n}\left[(-1)^{p} \frac{\mathrm{R} \pi}{2 g}+f_{0}^{\prime}\right] \frac{e^{i p \alpha}}{p^{2}} \sum_{1}^{p}\left[(-1)^{q} \frac{\mathrm{R} \pi}{2 g}+f_{0}^{\prime}\right] \frac{e^{i q \alpha}}{p+q}
$$

est donc elle aussi convergente dans l'intervalle ouvert $0, h$. Si la fonction $f\left(y_{0}\right)$ satisfait aux conditions limites, elle sera même convergente dans l'intervalle, extrémités comprises.

Quant à la série :

$$
\frac{2 k^{4} h}{g^{2} \pi} \sum_{1}^{n}\left[(-1)^{p} \frac{\mathrm{R} \pi}{2 g}+f_{0}^{\prime}\right] \frac{e^{i \nu \alpha}}{p^{2}} \sum_{1}^{n}\left[(-1) \frac{\mathrm{R} \pi}{2 g}+f_{0}^{\prime}\right] \frac{e^{i q \alpha}}{q^{2}(p+q)}
$$

on montrerait facilement qu'elle est absolument convergente.

d) Séries $\sum_{1}^{\infty} \sum_{1}^{\infty} V_{p q}=\sum_{1}^{\infty} \sum_{1}^{\infty} \frac{i a_{p} a_{q}\left(m_{p}+m_{q}\right)}{2 e^{2} \operatorname{sh} m_{p} h \operatorname{sh} m_{q} h}\left[\operatorname{ch}\left(m_{p}-m_{q}\right) y_{0}-\lambda_{p q} \operatorname{ch}\left(m_{p}+m_{q}\right) y_{0}\right]$

$$
\lambda_{p q}=-\frac{\left(\lambda_{p}-\lambda_{q}\right)^{2}+6 \lambda_{p} \lambda_{q}+\left(6 k^{4} / g^{2}\right)}{\left(\lambda_{p}-\lambda_{q}\right)^{2}+\left(4 k^{4} / g^{2}\right)}=-1+\frac{6 \lambda_{p} \lambda_{q}+\left(2 k^{4} / g^{2}\right)}{\left(\lambda_{p}-\lambda_{q}\right)^{2}+\left(4 k^{4} / g^{2}\right)}
$$

On calculera comme précédemment la limite de $\sum_{1}^{n} \sum_{1}^{n} V_{p q}$.

Cette série se décompose en deux produits de séries simples déjà étudiées ci-dessus, et en une somme qui constitue la partie réelle de :

$$
\sum_{1}^{n} \sum_{1}^{n} \frac{a_{p} a_{q}\left(\lambda_{p}+\lambda_{q}\right)}{2 e^{2} \sin \lambda_{p} \sin \lambda_{q} h} \frac{6 \lambda_{p} \lambda_{q}+\left(2 k^{4} / g^{2}\right)}{\left(\lambda_{p}-\lambda_{q}\right)^{2}+\left(4 k^{4} / g^{2}\right)} e^{i\left(\lambda_{p}+\lambda_{q}\right) y_{0}}
$$


Comme précédemment, on sommera sur la diagonale principale et dans la moitié du carré situé en dessous de la diagonale. Enfin on n'étudiera que le terme principal.

TERMES SITUÉS SUR LA DIAGONALE :

$$
\frac{3 g^{2}}{2 k^{4}} \sum_{1}^{n} \frac{\pi^{3}}{h^{3}}\left[(-1)^{n} \frac{\mathrm{R} \pi}{2 g}+f_{0}^{\prime}\right]^{2} \frac{e^{2 i p k x}}{p}+\frac{1}{2} \sum_{1}^{n}\left[(-1)^{p} \frac{\mathrm{R} \pi}{2 g}+f_{0}^{\prime}\right]^{2} \frac{e^{2 i p \alpha}}{p^{3}}
$$

Le deuxième terme converge absolument. Le premier se décompose en :

$$
\frac{3 g^{2}}{2 k^{4}} \frac{\pi^{3}}{h^{3}}\left[\frac{\mathrm{R}^{2} \pi^{2}}{4 g^{2}}+f^{\prime 2}{ }_{0}\right] \sum_{1}^{n} \frac{e^{2 i p \alpha}}{p}+\frac{3 g \pi^{4} \mathrm{R} f_{0}^{\prime}}{2 k^{4} h^{3}} \sum_{1}^{n} \frac{e^{-2 i p \beta}}{p}
$$

où l'on a posé :

$$
x=\frac{\pi y_{0}}{h} \quad \beta=\pi-\frac{2 \pi y_{0}}{h}
$$

Ces sommes ont des limites, sauf si $2 \alpha=0$ ou $2 \alpha=2 \pi$

$$
\left(y_{0}=0 \text { ou } y=h\right)
$$

et si $\beta=0 \quad\left(y_{0}=h / 2\right)$. Ainsi le milicu du batteur est également un point singulier au $2^{\circ}$ ordre d'approximation.

Termes situés me dessous de LA Diagonate :

Le terme principal s'écrit :

$$
\frac{\pi}{h} \sum_{p=2}^{n}\left[(-1)^{\prime} \frac{\mathrm{R} \pi}{2 g}+f_{0}^{\prime}\right] \frac{e^{i m k}}{p^{2}} \sum_{q=1}^{p-1}\left[(-1)^{q} \frac{\mathrm{R} \pi}{2 g}+f_{0}^{\prime}\right] e^{i q_{\alpha}} \frac{(p+q)}{q^{2}} \frac{6 p q+2\left(k^{4} h^{2}\right) /\left(g^{2} \pi^{2}\right)}{(p-q)^{2}+\left(4 k^{4} h^{2}\right) /\left(g^{2} \pi^{2}\right)}
$$

La fraction :

$$
\frac{p+q}{q^{2}} \frac{6 p q+2\left(k^{4} h^{2}\right) /\left(g^{2} \pi^{2}\right)}{(p-q)^{2}+\left(4 k^{4} h^{2}\right) /\left(g^{2} \pi^{2}\right)}
$$

peut se décomposer en éléments simples :

$$
\begin{gathered}
=\frac{2 \mathrm{~A}^{2} p}{p^{2}+4 \mathrm{~A}^{2}} \frac{1}{q^{2}}+\left[\frac{6}{p^{2}+4 \mathrm{~A}^{2}}+\frac{4 \mathrm{~A}^{2} p^{2}}{\left(p^{2}+4 \mathrm{~A}^{2}\right)^{2}}\right] \frac{1}{q}-\left[\frac{6 p^{2}+2 \mathrm{~A}^{2}}{p^{2}+4 \mathrm{~A}^{2}}+\frac{4 \mathrm{~A}^{2} p^{2}}{\left(p^{2}+4 \mathrm{~A}^{2}\right)^{2}}\right] \frac{q}{(p-q)^{2}+4 \mathrm{~A}^{2}} \\
+\left\lceil p \frac{18 p^{2}+26 \mathrm{~A}^{2}}{p^{2}+4 \mathrm{~A}^{2}}+\frac{8 \mathrm{~A}^{2} p^{3}}{\left(p^{2}+4 \mathrm{~A}^{2}\right)^{2}}\right] \frac{1}{(p-q)^{2}+4 \mathrm{~A}^{2}}
\end{gathered}
$$

où l'on a posé : $\mathrm{A}^{2}=\frac{k^{4} h^{2}}{g^{2} \pi^{2}}$

On en conclut que la somme des termes principaux se décompose en sommes de la forme:

$$
\begin{aligned}
& \sum_{:=2}^{n}\left[(-1)^{n} \frac{\mathrm{R} \pi}{2 g}+f_{0}^{\prime}\right] \frac{e^{i p \alpha}}{p^{3}} \sum_{1}^{p-1}\left[(-1)^{g} \frac{\mathrm{R} \pi}{2 g}+f_{0}^{\prime}\right] \frac{e^{i q \alpha}}{q^{2}} \\
& \sum_{:=}^{n}\left[(-1)^{p} \frac{\mathrm{R} \pi}{2 g}+f_{0}^{\prime}\right] \frac{e^{i p \alpha}}{p^{2}} \sum_{1}^{p-1}\left[(-1)^{g} \frac{\mathrm{R} \pi}{2 g}+f_{0}^{\prime}\right] \frac{e^{i q \alpha}}{q} \\
& \sum_{2}^{n}\left[(-1)^{p} \frac{\mathrm{R} \pi}{2 g}+f_{0}^{\prime}\right] \frac{e^{i \eta \mu}}{p^{2}} \sum_{1}^{n} \sum_{-}^{-1}\left[(-1)^{g} \frac{\mathrm{R} \pi}{2 g}+f_{0}^{\prime}\right] \frac{q e^{i q \mu}}{(p-q)^{2}+4 \mathrm{~A}^{2}} \\
& \sum_{2}^{n}\left[(-1)^{p} \frac{\mathrm{R} \pi}{2 g}+f_{0}^{\prime}\right] \frac{e^{i n \alpha}}{p} \sum_{1}^{p} \sum^{-1}\left[(-1)^{q} \frac{\mathrm{R} \pi}{2 g}+f_{0}^{\prime}\right] \frac{e^{i q \alpha}}{(p-q)^{2}+4 \mathrm{~A}^{2}}
\end{aligned}
$$

Dans la troisième et la quatrième sommes, on pose $r=p-q$. 
Elles se ramènent alors aux deux formes suivantes :

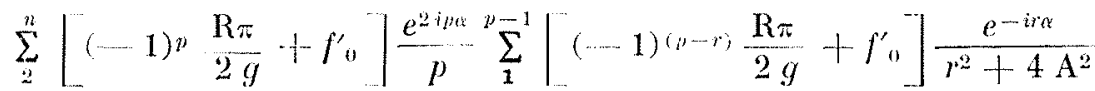

$$
\begin{aligned}
& -\sum_{\underline{2}}^{n}\left[(-1)^{p} \frac{\mathrm{R} \pi}{2 g}+f_{0}^{\prime}\right] \frac{e^{2 i p r}}{p^{2}} \sum_{1}^{p-1}\left|(-1)^{(n-r)} \frac{\mathrm{R} \pi}{2 g}+f_{0}^{\prime}\right| \frac{r e^{-i r \gamma}}{r^{2}+4 \mathrm{~A}^{2}}
\end{aligned}
$$

qui sont équivalentes à :

$$
\begin{aligned}
& \sum_{2}^{n}\left[(-1)^{p} \frac{\mathrm{R} \pi}{2 g}+f_{0}^{\prime}\right] \frac{e^{2 i p c} p-1}{p} \sum_{1}^{p}\left[(-1)^{(p-r)} \frac{\mathrm{R} \pi}{2 g}+f_{0}^{\prime}\right] \frac{e^{-i r \alpha}}{r^{2}} \\
\text { et : } \quad & \sum_{2}^{n}\left[(-1)^{n} \frac{\mathrm{R} \pi}{2 g}+f_{0}^{\prime}\right] \frac{e^{2 i p \alpha}}{p^{2}} \sum_{1}^{p-1}\left[(-1)^{(p-r)} \frac{\mathrm{R} \pi}{2 g}+f_{0}^{\prime}\right] \frac{e^{-i r^{2}}}{r}
\end{aligned}
$$

Les sommes [1] [2] et [4] sont de la forme :

$$
\sum_{p=2}^{\infty} \frac{\mathrm{S}_{p} e^{i p \alpha}}{p^{2}} \text { oul } \sum_{n=-2}^{\infty} \frac{\mathrm{S}_{p} e^{i p \alpha}}{p^{3}}
$$

$S_{p}$ étant les $p-1$ premiers termes d'une série absolument convergente [somme (1)] ou d'une série uniformément convergente $\left[\right.$ sommes (2) et (4)]. $S_{p}$ est donc bornée si $y_{0} \neq 0$ ou $y_{0} \neq h$. Les sommes (1), (2) et (4) peuvent donc être majorées par des séries absolument convergentes.

Quant à la somme (3), elle s'écrit sous la forme :

$$
\sum_{2}^{n}\left[\frac{\mathrm{R} \pi}{2 g}+(-1)^{p} f_{0}^{\prime}\right] \frac{e^{2 i p \alpha}}{p} \sum_{1}^{p}-^{1} \frac{\mathrm{R} \pi}{2 g} \frac{e^{-i r \alpha}}{r^{2}}+\sum_{2}^{n}\left[(-1)^{p} \frac{\mathrm{R} \pi}{2 g}+f_{0}^{\prime}\right] \frac{e^{2 i \nu \mu \alpha}}{p} \sum_{1}^{n-1} f_{0}^{\prime} \frac{e^{-i r \kappa}}{r^{2}}
$$

Les sommes :

$$
\mathrm{S}_{p}=\sum_{1}^{j-1} \frac{\mathrm{R} \pi}{2 g} \frac{e^{-i r_{\omega}}}{r^{2}} \quad \text { ou } \quad \mathrm{S}_{p}=\sum_{1}^{\mu-1} f_{0}^{\prime} \frac{e^{-i r \alpha}}{r^{2}}
$$

sont absolument convergentes. On peut donc écrire:

$$
\mathrm{S}_{p}=\mathrm{S}-\sum_{p}^{\infty} \frac{\mathrm{R} \pi}{2 g} \frac{e^{-i r \alpha}}{r^{2}}
$$

On peut évaluer l'ordre de grandeur du reste :

$$
\left|\frac{\mathrm{R} \pi}{2 g} \sum_{i}^{\infty} \frac{e^{-i r \alpha}}{r^{2}}\right|<\left|\frac{\mathrm{R} \pi}{2 g}\right| \int_{p-1}^{\infty} \frac{d r}{r^{2}}=\left|\frac{\mathrm{R} \pi}{2 g}\right| \mathrm{O}\left(\frac{1}{p}\right)
$$

d'où :

$$
\mathrm{S}-\left|\frac{\mathrm{R} \pi}{2 g}\right| \mathrm{O}\left(\frac{1}{p}\right) \leqslant S_{p} \leqslant \mathrm{~S}+\left|\frac{\mathrm{R} \pi}{2 g}\right| \mathrm{O}\left(\frac{1}{p}\right) \quad \mathrm{S}_{p}=\mathrm{S}-\mathrm{O}\left(\frac{1}{p}\right)
$$

Les sommes (3) sont donc finalement égales à :

$$
\mathrm{S} \sum_{i=}^{n}\left(\frac{\mathrm{R} \pi}{2 g}+(--1)^{p} f_{0}^{\prime}\right) \frac{e^{i p r}}{p}
$$

qui est uniformément convergente dans l'intervalle ouvert $0, h$, et :

$$
\left|\frac{\mathrm{R} \pi}{2 g}\right| \sum_{2}^{n}\left[\frac{\mathrm{R} \pi}{2 g}+(-1)^{p} f_{0}^{\prime}\right] \frac{\frac{e}{i p r}^{2}}{p^{2}}
$$

qui est absolument convergente.

En résumé, toutes les séries rencontrées convergent sur le batteur, sauf l'une d'elles qui diverge au point $h / 2$ si $\mathrm{R} \neq 0$ et $f_{0}^{\prime} \neq 0$. 
Si la fonction $f\left(y_{0}\right)$ satisfait aux conditions aux limites de fond et de surface, les séries sont absolument et uniformément convergentes dans tout l'intervalle fermé $o, h$.

Mais dans le cas général où $R \neq 0$ et $f_{0}^{\prime} \neq 0$, la convergence d'une partie des séries est simplement uniforme dans les intervalles ouverts $(0, h / 2)$ et $(h / 2, h)$.

Ces résultats nous permettent de déterminer la nature des singularités de $\xi\left(0, y_{0}, t\right)$ comme l'avait fait M. Biesel pour l'approximation au $1^{\text {er ordre. }}$

En effet, en utilisant les développements en série:

$1 /(1-u)=1+u+u^{2}+\ldots+u^{p}+\ldots$

$-\mathrm{L}(1-u)=u+\frac{u^{2}}{2}+\frac{u^{3}}{3}+\ldots+\frac{u^{p}}{p}+\ldots$

$-\int_{0}^{u} \mathrm{~L}(1-u) d u=(1-u) \mathrm{L}(1-u)=-u+\frac{u^{2}}{1 \times 2}+\frac{u^{3}}{2 \times 3}+\ldots+\frac{u^{p}}{p(p-1)}+\cdots$

dans lesquels on remplace $n$ par $e^{-\pi \tilde{x}_{0} / h}$, on obtient, après avoir séparé parties réelles et imaginaires :

$$
\begin{aligned}
& -\mathrm{L}\left(1-e^{-\pi \tilde{w}_{0} / h}\right)=\sum \frac{e^{-p \pi \tilde{n}_{0} / h}}{p}=\sum e^{-\mu \pi x_{1} / h} \frac{\cos p \pi y_{0} / h}{p}-i \sum e^{-p \pi x_{0} / h} \frac{\sin p \pi y_{0} / h}{p} \\
& \left(1-e^{-\pi \tau_{\mathrm{i}} / h}\right) \mathbf{L}\left(1-e^{-\pi \tau_{\mathrm{o}} / h}\right)=-e^{-\pi z_{0} / h}+\sum e^{-p \pi z_{\mathrm{i}} / h} \frac{\cos p \pi y_{0} / h}{p(p-1)}-i \sum e^{-j \pi \pi_{0} / h} \frac{\sin p \pi y_{0} / h}{p(p-1)}
\end{aligned}
$$

En remplaçant les infiniment petits par des quantités équivalentes, il vient:

$$
\begin{aligned}
& -\mathrm{L} \frac{\pi z_{0}}{h} \sim \sum e^{-p \pi x_{0} / h} \frac{\cos p \pi y_{0} / h}{p}-i \sum e^{-p \pi x_{0} / h}-\frac{\sin }{p \pi y_{0} / h} \\
& p \\
& 1+\frac{\pi z_{0}}{h} \mathrm{~L} \frac{\pi z_{0}}{h} \sim \sum e^{-m \pi x_{0} / h} \frac{\cos p \pi y_{0} / h}{p^{2}}-i \sum e^{-\mu \pi x_{0} / h}-\frac{\sin }{p^{2}} \frac{p \pi y_{0} / h}{p^{2}}
\end{aligned}
$$

On en conclut que la fonction $\zeta\left(x_{0}, y_{0}, t\right) \ldots i \eta_{1}\left(x_{0}, y_{0}, t\right)$, dont la partie réelle se réduit sur le batteur à une combinaison de séries de la forme:

$$
\sum \frac{\cos p \pi y_{0} / h}{p} \quad \text { ou } \sum \frac{\cos p \pi y_{0} / h}{p^{2}}
$$

ou de produits :

$$
\left\{\left(\sum \frac{\cos p \pi y_{0} / h}{p}\right)\left(\sum \frac{\cos p \pi y_{0} / h}{p^{2}}\right)\right\}
$$

possède des singularités de la forme $\log z_{0}, z_{0}\left(\log z_{0}\right), z_{0}\left(\log z_{0}\right)^{2}$ et des termes d'ordre supérieur.

Ces singularités sont faibles, et $\xi\left(0, y_{0}, t\right)$ est de carré intégrable sur l'intervalle $0, h$.

Etude a priori des singularités des fonctions $\mathrm{X}_{22}$ ET $\mathrm{Y}_{2}$.

Les fonctions $X_{2}$ et $Y_{2}$ sont solutions dun système d'équations dont les coefficients comportent des singularités, puisque les seconds membres s'expriment au moyen des fonctions $\mathrm{X}_{1}$ et $Y_{1}$.

La solution particulière du système complet possèdera donc des singularités (engendrées par celles de $X_{1}$ et $Y_{1}$ ). Nous allons tout d'abord rechercher celles-ci.

La solution générale des équations sans second membre vérifie d'autre part des équations dont les coefficients sont réguliers dans tout le domaine. Mais elle est assujettie à des conditions frontières qui peuvent, comme pour le premier ordre, introduire des singularités que nous devrons également rechercher. On connaîtra alors toutes les singularités de la solution. 


\section{Etude de la solution particulitire.}

Dans le premier chapitre, on a étudié les singularités des fonctions $\mathrm{X}_{1}\left(x_{0}, y_{0}\right)$ et $\mathrm{Y}_{1}\left(x_{0}, y_{0}\right)$. Celles-ci sont liées à la solution du $1^{\text {tr }}$ ordre par les équations :

$$
\begin{gathered}
\mathrm{x}_{1}\left(x_{0}, y_{0}, t\right)=-\frac{a_{0}}{e} \frac{\operatorname{ch} m_{0} y_{0}}{\operatorname{sh} m_{0} h} \sin m_{0} x_{0} \cos k t+\mathrm{X}_{1}\left(x_{0}, y_{0}\right) \sin k t \\
\mathrm{Y}_{1}\left(x_{0}, y_{0}, t\right)=\frac{a_{0}}{e} \frac{\operatorname{sh~} m_{0} y_{0}}{\operatorname{sh} m_{0} h} \cos m_{0} x_{0} \cos k t+\mathrm{Y}_{1}\left(x_{0}, y_{0}\right) \sin k t
\end{gathered}
$$

Les équations du mouvement peuvent donc s'écrire :

$$
\begin{gathered}
\left.\frac{\partial \mathrm{X}_{2}}{\partial x_{0}}+\frac{\partial \mathbf{Y}_{2}}{\partial y_{0}}=-\frac{\mathrm{D}\left[\mathrm{X}_{1}\left(x_{0}, y_{0}, t\right), \mathrm{Y}_{1}\left(x_{0}, y_{0}, t\right)\right]}{\mathrm{D}\left(x_{0}, y_{0}\right)}\right) \\
=-\frac{\mathrm{D}\left[\mathrm{X}_{1}\left(x_{0} y_{0}\right), \mathrm{Y}_{1}\left(x_{0}, y_{0}\right)\right]}{\mathrm{D}\left(x_{0}, y_{0}\right)} \sin ^{2} k t-\frac{a_{0} m_{0}}{2 e} \frac{\operatorname{ch} m_{0} y_{0}}{\operatorname{sh} m_{0} h} \cos m_{0} x_{0} \sin 2 k t\left(\frac{\partial \mathrm{X}_{1}}{\partial x_{0}}-\frac{\partial \mathrm{Y}_{1}}{\partial y_{0}}\right)
\end{gathered}
$$

$-\frac{a_{0} m_{0}}{2 e} \frac{\operatorname{sh} m_{0} y_{0}}{\operatorname{sh} m_{0} h} \sin m_{0} x_{0} \sin 2 k t\left(\frac{\partial \mathrm{X}_{1}}{\partial y_{0}}+\frac{\partial \mathrm{Y}_{1}}{\partial x_{0}}\right)+\frac{a_{0}{ }^{2} m_{0}{ }^{2}}{2 e^{2} \operatorname{sh}^{2} m_{0} h}\left(\cos 2 m_{0} x_{0}+\operatorname{ch} 2 m_{0} y_{0}\right) \cos ^{2} k t$

On peut remplacer d'ailleurs $\mathrm{X}_{1}$ et $\mathrm{Y}_{1}$ par les développements limités valables au pied du batteur :

$$
\begin{gathered}
\mathrm{X}_{1}=-\frac{\mathrm{R}}{g}\left(x_{0} \log 9-0 y_{0}\right)+f_{0}+\mathrm{B} x_{10}-\mathrm{C} y_{0}+\mathrm{P}_{2}+\mathrm{P}_{3}+\ldots \\
\mathrm{Y}_{1}=\frac{\mathrm{R}}{g}\left(y_{0} \log \rho+0 x_{0}\right)+\mathrm{A}-\mathrm{C} x_{0}-\mathrm{B} y_{0}-\mathrm{Q}_{2}-\mathrm{Q}_{3} \ldots
\end{gathered}
$$

On obtient ainsi :

$$
\frac{\partial \mathrm{X}_{2}}{\partial x_{0}}+\frac{\partial \mathrm{Y}_{2}}{\partial y_{0}}=\sin ^{2} k t\left\{\begin{array}{l}
\frac{\mathrm{R}^{2}}{g^{2}}\left[(\log 0)^{2}+0^{2}\right]+\frac{2 \mathrm{R}}{g}\left(\frac{\mathrm{R}}{g}-\mathrm{B}\right) \log 0-\frac{2 \mathrm{RC}}{g} \\
-\frac{\mathrm{R}}{g} \log \rho\left[\frac{\partial \mathrm{P}_{2}}{\partial x_{0}}+\frac{\partial \mathrm{Q}_{2}}{\partial y_{0}}+\frac{\partial \mathrm{P}_{3}}{\partial x_{0}}+\frac{\partial \mathrm{Q}_{3}}{\partial y_{0}}+\ldots . .\right] \\
+\frac{\mathrm{R} 0}{g}\left[\frac{\partial \mathrm{P}_{2}}{\partial y_{0}}-\frac{\partial \mathrm{Q}_{2}}{\partial x_{0}}+\frac{\partial \mathrm{P}_{3}}{\partial y_{0}}-\frac{\partial \mathrm{Q}_{3}}{\partial x_{0}}+\ldots . .\right] \\
+\frac{\partial \mathrm{P}_{2}}{\partial x_{0}} \frac{\partial \mathrm{Q}_{2}}{\partial y_{0}}-\frac{\partial \mathrm{P}_{2}}{\partial y_{0}} \frac{\partial \mathrm{Q}_{2}}{\partial x_{0}}+\frac{\partial \mathrm{P}_{3}}{\partial x_{0}} \frac{\partial \mathrm{Q}_{3}}{\partial y_{0}}-\frac{\partial \mathrm{P}_{3}}{\partial y_{0}} \frac{\partial \mathrm{Q}_{3}}{\partial x_{0}}+\ldots . .
\end{array}\right.
$$

$-\frac{m_{0} a_{0}}{2 e \operatorname{sh} m_{0} h} \sin 2 k t\left[-\frac{2 \mathrm{R}}{g} \log \rho \operatorname{ch} m_{0} y_{0} \cos m_{0} x_{0}+\frac{2 \mathrm{R} \theta}{g} \operatorname{sh~} m_{0} y_{0} \sin m_{0} x_{0}\right]$

$$
+\frac{a_{0}^{2} m_{0}^{2}}{2 e^{2} \operatorname{sh}^{2} m_{0} h}\left(\cos 2 m_{0} x_{0}+\operatorname{ch} 2 m_{0} y_{0}\right) \cos ^{2} k t
$$

$$
\frac{\partial \mathrm{X}_{2}}{\partial y_{0}}-\frac{\partial \mathrm{Y}_{2}}{\partial x_{0}}=0
$$

Pour établir ces équations, on a tenu compte du fait que $P_{2}$ et $Q_{2}, P_{3}$ et $Q_{3}$, etc, sont harmoniques conjugués. On a donc

$$
\frac{\partial \mathrm{P}_{2}}{\partial x_{0}}-\frac{\partial \mathrm{Q}_{2}}{\partial y_{0}}=0 \quad \frac{\partial \mathrm{P}_{2}}{\partial y_{0}}+\frac{\partial \mathrm{Q}_{2}}{\partial x_{0}}=0
$$

On a d'ailleurs :

$$
\begin{aligned}
& \mathrm{P}_{2}=a_{2}\left(x_{0}{ }^{2}-y_{0}{ }^{2}\right) \quad \mathrm{P}_{3}=a_{3}\left(x_{0}{ }^{3}-3 x_{0} y_{0}{ }^{2}\right) \\
& \mathrm{Q}_{2}=2 a_{2} x_{0} y_{0} \quad \mathrm{Q}_{3}=a_{3}\left(3 x_{0}{ }^{2} y_{0}-y_{0}{ }^{3}\right) \\
& \operatorname{ch~} m_{0} y_{0} \cos m_{0} x_{0}=1-\left(\mathrm{m}_{0}{ }^{2} / 2\right)\left(x_{0}{ }^{2}-y_{0}{ }^{2}\right) \\
& \operatorname{sh~} m_{0} y_{0} \sin m_{0} x_{0}=m_{0}{ }^{2} x_{0} y_{0}
\end{aligned}
$$


d'où le système d'équations :

$$
\begin{aligned}
& \frac{\partial X .}{\partial y_{10}}-\frac{\partial Y_{2}}{\partial x_{0}}=0
\end{aligned}
$$

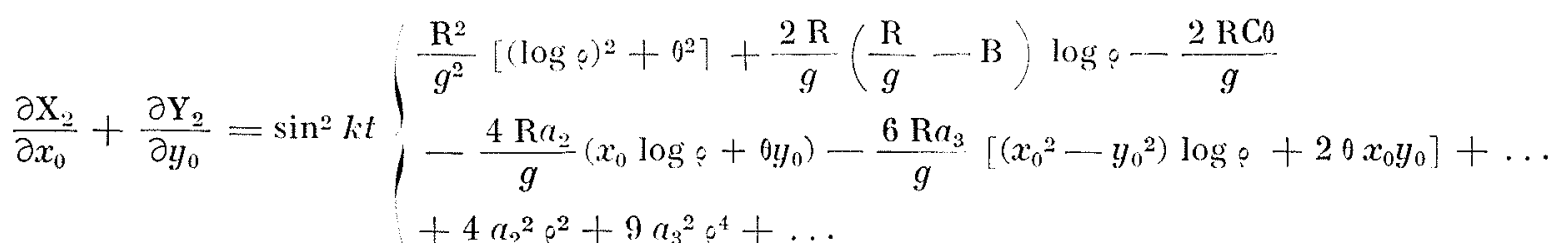

$$
\begin{aligned}
& \left.-\frac{m_{0} a_{0}}{2 e \operatorname{sh} m_{0} h} \sin 2 k t ;-\frac{2 \mathrm{R}}{g} \log \rho+\frac{\mathrm{R} m_{0}^{2} a_{3}}{g}\left[\left(x_{0}^{2}-y_{0}^{2}\right) \log \rho+2 \theta x_{0} y_{0}\right]+\ldots\right\} \\
& +\frac{a_{0}^{2} m_{0}}{2 e^{2} \operatorname{sh}^{2} m_{0} h}\left(\cos 2 m_{0} x_{0}+\operatorname{ch} 2 m_{0} y_{0}\right) \cos ^{2} k t
\end{aligned}
$$

Il est facile de trouver une solution particulière de ce système sous la forme de sommes de solutions particulières. On obtient ainsi:

$$
\begin{aligned}
& \frac{\mathrm{R}^{2}}{2 g^{2}}\left[x_{0}(\log \rho)^{2}-x_{0} \log _{?}+x_{0} 0^{2}-y_{0} 0\right]+\frac{\mathrm{R}}{2 g}\left(\frac{\mathrm{R}}{g}-\mathrm{B}\right)\left(2 x_{0} \log ?-x_{0}\right) \\
& \mathrm{X}_{2}^{\prime}=\sin ^{2} k t \mid-\frac{\mathrm{RC}}{2 g}\left(2 \theta x_{0}-y_{0}\right)-\frac{\mathrm{R} a_{2}}{g}\left(2 x_{0}{ }^{2} \log \rho-\rho^{2}\right) \\
& -\frac{\mathrm{R} a_{3}}{2 g}\left(4 x_{0}{ }^{3} \log \varphi+2 y_{0}^{3} 0+6 x_{0}^{2} y_{0} \theta-3 x_{0} y_{0}^{2}-3 x_{0}^{3}\right)+\mathrm{O}\left(\rho^{4} \log \rho\right)+\mathrm{O}\left(\mathrm{p}^{4} \theta\right) \\
& +\frac{4 a_{2}^{2} x_{0}{ }^{3}}{3}+\frac{9 a_{3}^{2} x_{0}^{5}}{5} \\
& +\frac{\mathrm{R} a_{0} m_{0} \sin 2 k t}{4 g e \operatorname{sh} m_{0} h}\left\{\begin{array}{l}
a_{2}\left(2 x_{0} \log \varphi-x_{0}\right) \\
-\frac{m_{0}{ }^{2} a_{3}}{6}\left(4 x_{0}{ }^{3} \log 0+2 y_{0}{ }^{3} 0+6 x_{0}{ }^{2} y_{0} \theta-3 x_{0} y_{0}{ }^{2}-3 x_{0}{ }^{3}\right)+o\left(0^{4} \log \varphi\right)+o\left(\varphi^{4} \theta\right)
\end{array}\right. \\
& +\frac{a_{0}^{2} \cos ^{2} k t}{4 e^{2} \operatorname{sh}^{2} m_{0} h} \sin 2 m_{0} x_{0}
\end{aligned}
$$

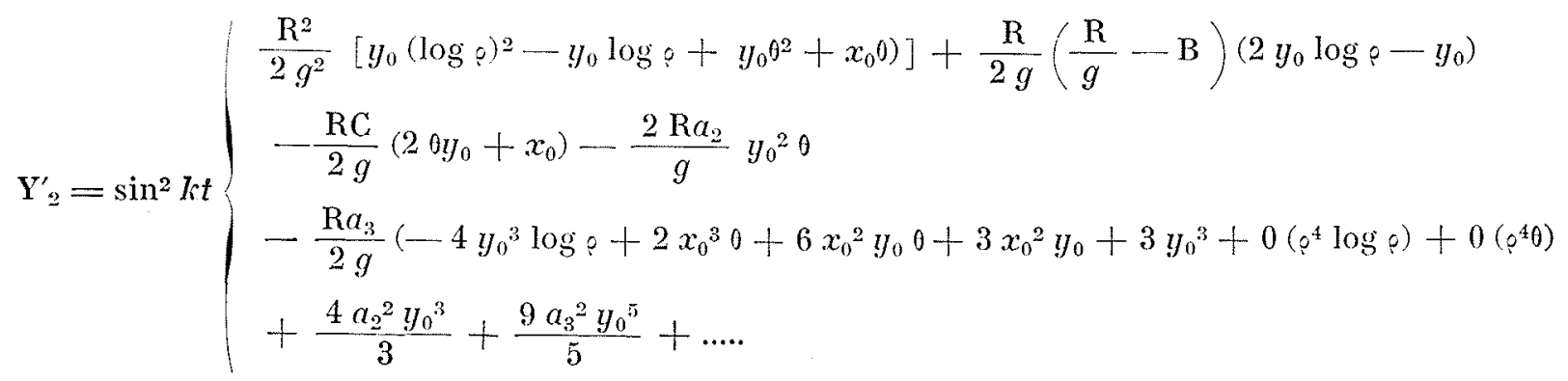$$
+\frac{\mathrm{R} a_{0} m_{0} \sin 2 k t}{4 g e \operatorname{sh} m_{0} h}\left\{\begin{array}{l}
a_{2}\left(2 y_{0} \log \rho-y_{0}\right) \\
-\frac{m_{0}{ }^{2} a_{3}}{6}\left(-4 y_{0}^{3} \log \rho+2 x_{0}^{3} \theta+6 x_{0} y_{0}{ }^{2} \theta+3 x_{0}^{2} y_{0}+3 y_{0}^{2}\right)+O\left(\rho^{4} \log \rho\right)+\mathrm{O}\left(\rho^{4} \theta\right)
\end{array}\right.
$$$$
+\frac{a_{0}^{2} \cos ^{2} k t}{4 e^{2} \operatorname{sh}^{2} m_{0} h} \operatorname{sh} 2 m_{0} y_{0}
$$

Etude de la solution Générale de L'ÉQUation SANS SECOND MEMBRe.

Soit $\mathrm{X}_{2}^{\prime \prime}, \mathrm{Y}^{\prime \prime}{ }_{2}$ la solution générale de l'équation sans $2^{\mathrm{e}}$ membre. Comme dans la théorie au $1^{\text {er }}$ ordre $\mathrm{X}_{2}^{\prime \prime}$ et $-\mathrm{Y}^{\prime \prime}{ }_{2}$ sont harmoniques conjuguées et la fonction $\mathrm{F}\left(z_{0}\right)=\mathrm{X}_{2}^{\prime \prime}-i \mathrm{Y}^{\prime \prime}{ }_{2}$ est analytique dans le domaine étudié. 
La solution du problème du batteur est :

$$
\mathrm{X}_{2}=\mathrm{X}_{2}^{\prime}+\mathrm{X}_{2}^{\prime \prime} \quad \mathrm{Y}_{2}=\mathrm{Y}_{2}^{\prime}+\mathrm{Y}_{2}^{\prime \prime}
$$

$\mathrm{X}_{2}$ et $\mathrm{Y}_{2}$ étant assujetties à des conditions limites au fond, en surface, à l'infini et sur le batteur.

Les trois premières s'expriment par des équations dont les coefficients sont réguliers sur chacune de ces limites. $\mathrm{X}_{2}{ }_{2}$ et $\mathrm{Y}_{2}^{\prime \prime}{ }_{2}$ ne peuvent donc y avoir de singularités. De même, si $f\left(y_{0}\right)$ est régulière dans l'intervalle ouvert, $0<y_{0}<h, \mathrm{X}^{\prime \prime}$ : et $\mathrm{Y}^{\prime \prime}{ }_{2}$ sont régulières sur cet intervalle.

Mais aux points d'angle (extrémités du batteur) les fonctions doivent satisfaire simultanément à deux conditions limites différentes (en général). Elles doivent donc prendre des valeurs différentes selon le chemin parcouru lorsqu'on approche de ces points (paroi du batteur d'une part, fonds ou surface libre d'autre part). Ainsi les extrémités du batteur sont des points critiques.

\section{A) Étude du point situé au pied du batteur}

Comme dans la théorie au $1^{\mathrm{er}}$ ordre, on transforme le point d'angle en un point ordinaire du contour, en prolongeant analytiquement les fonctions $\mathrm{X}_{2}{ }_{2}$ et $\mathrm{Y}^{\prime \prime}{ }_{2}$ dans la bande

$$
-h \leqslant y_{0} \leqslant 0 \quad x_{0} \geqslant 0 .
$$

On considère pour cela le mouvement image ${ }^{*}$ ) du mouvement réel, à travers le fond du canal et l'on supprime la paroi constituant le fond.

La fonction harmonique $\mathrm{X}_{2}^{\prime \prime}$ est définie dans tout le domaine $-h \leqslant y_{0} \leqslant+h \quad x_{0} \geqslant 0$. Elle est déterminée par les conditions de surface $\left.\left(\partial^{2} \mathrm{X}^{2} / \partial t^{2}\right)+g\left(\partial \mathrm{X}_{2} / \partial y_{0}\right)=0 \quad y_{0}= \pm h\right)$, la condition à l'infini et la condition sur le batteur.

$$
\mathrm{X}_{2}^{\prime \prime}\left(0, \eta_{0}, t\right)=\mathrm{Y}_{1}\left(0, \eta_{0}, t\right) \frac{d f\left(y_{0}\right)}{d y_{0}} \sin k t-\mathrm{X}_{2}^{\prime}\left(0, y_{0}, t\right)
$$

soit :

$$
X^{\prime \prime}{ }_{2}\left(0, y_{0}, t\right)=\frac{a_{0} \operatorname{sh} m_{0} y_{0}}{2 e \operatorname{sh} m_{0} h} \frac{d f\left(y_{0}\right)}{d y_{0}} \sin 2 k t+\mathrm{Y}_{1}\left(0, y_{0}\right) \frac{d f\left(y_{0}\right)}{d y_{0}} \sin ^{2} k t-\mathrm{X}_{2}^{\prime}\left(0, y_{0}, t\right)
$$

La fonction harmonique conjuguée $\mathrm{Y}^{\prime \prime}{ }_{2}$ se déduit de la fonction $\mathrm{X}_{2}{ }_{2}$ à une constante additive près. Lorsqu'on parconrt l'axe $0, y_{0}$ en traversant l'origine, $\mathrm{X}_{2}{ }_{2}$ est continu et s'annule pour $y_{0}=0$. Par contre, $\partial \mathrm{X}^{\prime \prime}{ }_{2} / \partial y_{0}$ présente des discontinuités.

On les calculera en remplaçant $\mathrm{Y}_{1}$ par le développement limité valable au pied du batteur $\left[\mathrm{A}=\mathrm{C}=0 \quad(\mathrm{R} / g)=2\left(f_{0}^{\prime} / \pi\right)\right] \quad \mathrm{B}=\left(2 f_{0}^{\prime} / \pi\right)+a_{0}:$

$$
\begin{aligned}
& \left.\frac{\partial \mathrm{X}_{0}^{\prime \prime}}{\partial y_{0}}\right|_{x_{0}=0}=\frac{a_{0}}{2 e \operatorname{sh} m_{0} h} \sin 2 k t\left[m_{0} \operatorname{ch} m_{0} y_{0} \frac{d f\left(y_{0}\right)}{d y_{0}}+\operatorname{sh} m_{0} y_{0} \frac{d^{2} f\left(y_{0}\right)}{d y_{0}{ }^{2}}\right\rceil \\
& +\left[\frac{2 f_{0}^{\prime}}{\pi} \log \left|y_{0}\right|-a_{0}+3 a_{3} y_{0}{ }^{2} \cdots\right] \frac{d f\left(y_{0}\right)}{d y_{0}} \sin ^{2} k t \\
& +\left[\frac{2 f_{0}^{\prime}}{\pi} y_{0} \log \left|y_{0}\right| \cdots\left(\frac{2 f_{0}^{\prime}}{\pi}+a_{0}\right) y_{0}+a^{3} y_{0}{ }^{3}+a_{0}\right] \frac{d^{2} f\left(y_{0}\right)}{d y_{0}{ }^{2}} \sin ^{2} k t \\
& -\left[-\frac{2 f_{0}^{\prime 2}{ }^{2}}{\pi^{2}}+\frac{4 f_{0}^{\prime}}{\pi} a_{2} y_{0}-\frac{6 f_{0}^{\prime}}{\pi} a_{3} \theta y_{0}{ }^{2}\right] \sin ^{2} k t+\frac{f_{0}^{\prime} a_{0} a_{3} m_{0}^{3}}{2 \pi e \operatorname{sh} m_{0} h} y_{0}^{2} \theta \sin 2 k t
\end{aligned}
$$

On a des discontinuités de première et deuxième espèces à l'origine :

$$
\left(\frac{\partial X^{\prime \prime}}{\partial y_{0}}\right)_{\left.y_{0}=+1\right)}--\left(\frac{\partial X_{2}^{\prime \prime}}{\partial y_{0}}\right)_{y_{0}=-0}=\left(\frac{4 f_{0}^{\prime}{ }^{2}}{\pi} \log \left|y_{0}\right|-2 \alpha_{0} f_{0}^{\prime}+\frac{2 f_{0}^{\prime 2}}{\pi}\right) \sin ^{2} k t+\frac{a_{0} m_{0} f_{0}^{\prime}}{e \operatorname{sh} m_{0} h} \sin 2 k t
$$

${ }^{*}$ Le prolongement analytique est possible puisque $\mathrm{Y}_{2}^{\prime \prime}=0$ sur le fond. 

fonction :

Dans le voisinage de l'origine, $\partial \mathrm{X}^{\prime \prime}{ }_{2} / \partial y_{0}$ se comporte donc comme la partie réelle de la $g\left(z_{0}\right)=-\frac{2 i f_{0}^{\prime}}{\pi}\left(\frac{f_{0}^{\prime}}{\pi}-a_{0}\right) \log z_{0} \sin ^{2} k t-\frac{2 i f_{0}^{\prime}{ }^{2}}{\pi^{2}}\left(\log z_{0}\right)^{2} \sin ^{2} k t-\frac{i a_{0} m_{0} f_{0}^{\prime}}{e \pi \operatorname{sh} m_{0} h} \log z_{0} \sin 2 k t$

En retranchant la singularité, on obtient :

$\mathfrak{a} ; i \frac{d \mathrm{~F}\left(z_{0}\right)}{d z_{0}}+i\left[\frac{2 f_{0}^{\prime}}{\pi}\left(\frac{f_{0}^{\prime}}{\pi}-a_{0}\right) \log z_{0}+\frac{2 f_{0}^{\prime}{ }^{2}}{\pi^{2}}\left(\log z_{0}\right)^{2}\right] \sin ^{2} k t+\frac{i a_{0} m_{0} f_{0}^{\prime}}{\pi e \operatorname{sh} m_{0} h} \log z_{0} \sin ^{2} k t^{\prime}$

$$
\begin{aligned}
& \left.=\left.\frac{a_{0} m_{0} \sin 2 k t}{2 e \operatorname{sh} m_{0} h}\right|_{0} ^{-} y_{0} \frac{d^{2} f\left(y_{0}\right)}{d y_{0}{ }^{2}}+\frac{m_{0}{ }^{2} y_{0}{ }^{2}}{2} \frac{d f\left(y_{0}\right)}{d y_{0}}+\frac{f_{0}^{\prime} a_{3} m_{0}{ }^{2}}{\pi} y_{0}{ }^{2} \theta+f^{\prime \prime}{ }_{0} \mathrm{O}\left(y_{0}{ }^{3}\right)\right] \\
& +\sin ^{2} k t\left[\frac{2 f_{0}^{\prime}}{\pi} y_{0} \log \left|y_{0}\right| \frac{d^{2} f\left(y_{0}\right)}{d y_{0}{ }^{2}}-\left(\frac{2 f_{0}^{\prime}}{\pi}+a_{0}\right) y_{0} \frac{d^{2} f\left(y_{0}\right)}{d y_{0}{ }^{2}}-\frac{4 f_{0}^{\prime} a_{2}}{\pi} y_{0}+\right. \\
& \left.+3 a_{3} \frac{d f\left(y_{0}\right)}{d y_{0}} y_{0}{ }^{2}+\frac{6 a_{3} f_{0}^{\prime} \theta y_{0}{ }^{2}}{\pi}+f_{0}^{\prime} \mathrm{O}\left(y_{0}^{3}\right)\right]
\end{aligned}
$$

Cette expression se comporte à son tour comme la partie réelle de la fonction :

$$
\begin{gathered}
\frac{a_{0} m_{0} \sin 2 k t}{2 e \operatorname{sh} m_{0} h}\left[\frac{i m_{0}{ }^{2} f_{0}^{\prime}}{\pi} z_{0}{ }^{2} \log z_{0}+\frac{i f_{0}^{\prime} a_{3} m_{0}{ }^{2}}{\pi} z_{0}{ }^{2} \log z_{0}-i \mathrm{E}-6 z_{0}{ }^{2}-i z_{0} f^{\prime \prime}{ }_{0}+f^{\prime \prime}{ }_{0} \mathrm{O}\left(z_{0}{ }^{3} \log z_{0}\right)\right] \\
+\sin ^{2} k t\left[-\frac{2 i f_{0}^{\prime} f^{\prime \prime}{ }_{0}}{\pi} z_{0} \log z_{0}+i\left(\frac{2 f_{0}^{\prime}}{\pi}+a_{0}\right) f^{\prime \prime} z_{0} z_{0}+\frac{4 i f_{0}^{\prime} a_{2} z_{0}}{\pi}+i \mathrm{~F}_{0}+\mathrm{H} z_{0}{ }^{2}\right. \\
\left.+\frac{12 i a_{3} f_{0}^{\prime} z_{0}{ }^{2}}{\pi} \log z_{0}+f_{0}^{\prime} \mathrm{O}\left(z_{0}{ }^{3} \log z_{0}\right)\right]
\end{gathered}
$$

On a donc le développement limité :

$$
\begin{aligned}
& \frac{d \mathrm{~F}\left(z_{0}\right)}{d z_{0}}=\sin ^{2} k t\left\{\begin{array}{l}
-\frac{2 f_{0}^{\prime}}{\pi}\left(\frac{f_{0}^{\prime}}{\pi}-a_{0}\right) \log z_{0}-\frac{2 f_{0}^{\prime}}{\pi^{2}}\left(\log z_{0}\right)^{2}-\frac{2 f_{0}^{\prime} f_{0}^{\prime \prime}}{\pi} z_{0} \log z_{0}+\left(\frac{2 f_{0}^{\prime}}{\pi}+a_{0}\right) f^{\prime \prime} z_{0} \\
+\frac{4 f_{0}^{\prime} a_{2} z_{0}}{\pi}+\frac{12 a_{3} f_{0}^{\prime}}{\pi} z_{0}^{2} \log z_{0}+\mathrm{F}_{0}-i \mathrm{H} z_{0}^{2}+f_{0}^{\prime} \mathrm{O}\left(z_{0}^{3} \log z_{0}\right)
\end{array}\right. \\
& -\frac{a_{0} m_{0}}{2 e \operatorname{sh} m_{0} h} \sin 2 k t\left(\frac{2 f_{0}^{\prime}}{\pi} \log z_{0}-\left(\frac{m_{0}^{2} f_{0}^{\prime}}{\pi}+\frac{m_{0}{ }^{2} f_{0}^{\prime} a_{3}}{\pi}\right) z_{0}{ }^{2} \log z_{0}+z_{0} f_{0}^{\prime \prime}+\mathrm{E}_{0}-i \mathrm{G} z_{0}{ }^{2}+\mathrm{O}\left(z_{0}{ }^{3} \log z_{0}\right)\right)
\end{aligned}
$$

En intégrant, il vient :

$$
\begin{aligned}
& \mathrm{F}\left(z_{0}\right)=\sin ^{2} k t\left\{\begin{array}{l}
-\frac{2 f_{0}^{\prime}}{\pi}\left(\frac{f_{0}^{\prime}}{\pi}-a_{0}\right)\left(z_{0} \log z_{0}-z_{0}\right)-\frac{2 f_{0}^{\prime}{ }^{2}}{\pi^{2}} z_{0}\left(\log z_{0}\right)^{2}+\frac{4 f_{0}^{\prime}}{\pi^{2}}\left(z_{0} \log z_{0}-z_{0}\right) \\
-\frac{2 f_{0}^{\prime} f_{0}^{\prime \prime}}{\pi}\left(\frac{z_{0}{ }^{2}}{2} \log z_{0}-\frac{z_{0}{ }^{2}}{4}\right)+\left[\left(\frac{2 f_{0}^{\prime}}{\pi}+a_{0}\right) f^{\prime \prime}{ }_{0}+\frac{4 a_{2} f_{0}^{\prime}}{\pi}\right] \frac{z_{0}{ }^{2}}{2} \\
+f_{0}^{\prime} \mathrm{O}\left(z_{0}{ }^{4} \log z_{0}\right)+\mathrm{F}_{0} z_{0}-\frac{\mathrm{i} H z_{0}{ }^{3}}{3}+\mathrm{I}+\frac{12 a_{3} f_{n}^{\prime}}{\pi}\left(\frac{z_{0}{ }^{3}}{3} \log z_{0}-\frac{z_{0}{ }^{3}}{9}\right)
\end{array}\right. \\
& -\frac{a_{0} m_{0} \sin 2 k t}{2 e \operatorname{sh} m_{0} h}, \frac{2 f_{0}^{\prime}}{\pi}\left(z_{0} \log z_{0}-z_{0}\right)-\frac{m_{0}^{2} f_{0}^{\prime}}{\pi}\left(1+a_{3}\right)\left(\frac{z_{0}^{3} \log z_{0}}{3}-\frac{z_{0}{ }^{3}}{9}\right)+\frac{z_{0}{ }^{2} f_{0}^{\prime \prime}}{2} \\
& \left.+\mathrm{E}_{0} z_{0}-\frac{i \mathrm{G} z_{0}^{3}}{3}+\mathrm{J}+\mathrm{O}\left(z_{0}^{4} \log z_{0}\right)\right\}+\mathrm{K}
\end{aligned}
$$

Pour le batteur plan qui nous intéresse plus spécialement $f^{\prime \prime}{ }_{0}=0$. On a donc, en séparant les parties réelles et imaginaires : 
$\mathrm{x}_{2}=\sin ^{2} k t\left\{\begin{array}{l}\frac{2 f_{0}^{\prime}}{\pi}\left(\frac{f_{0}^{\prime}}{\pi}+a_{0}\right)\left(x_{0} \log \rho-\theta y_{0}-x_{0}\right)-\frac{2 f_{0}^{\prime}{ }^{2}}{\pi^{2}}\left\lceil x_{0}(\log \rho)^{2}-x_{0} \theta^{2}-2 \theta y_{0} \log \vartheta\right\rceil \\ +\frac{2 f_{0}^{\prime} a_{2}}{\pi}\left(x_{0}^{2}-y_{0}^{2}\right)+\mathrm{I}_{1}+\mathrm{F}_{0} x_{0}+\mathrm{O}\left(\rho^{3} \log \rho\right)\end{array}\right.$

$-\frac{\alpha_{0} m_{0} \sin 2 k t}{2 e \operatorname{sh} m_{0} h}, \frac{2 f_{0}^{\prime}}{\pi}\left(x_{11} \log \rho-\theta y_{0}-x_{0}\right)+\mathrm{E}_{t} x_{1}+\mathrm{J}_{1}+O\left(\rho_{0} \log \rho\right) i+\mathrm{K}_{1}$

$Y_{2}=-\sin ^{2} k t\left\{\begin{array}{l}\frac{2 f_{0}^{\prime}}{\pi}\left(\frac{f_{0}^{\prime}}{\pi}+a_{0}\right)\left(y_{0} \log _{\rho}+0 x_{0}-y_{0}\right)-\frac{2 f_{0}^{\prime}{ }^{2}}{\pi^{2}}\left[y_{0}(\log \rho)^{2}-y_{0} \theta^{2}+2 \theta x_{0} \log \rho\right] \\ +\frac{4 f_{0}^{\prime} a_{2}}{\pi} x_{0} y_{0}+F_{0}^{\prime} y_{0}+I_{2}+O\left(\rho^{3} \log \rho\right)\end{array}\right.$

$+\frac{a_{0} m_{0} \sin 2 k t}{2 e \operatorname{sh} m_{0} h}\left[\frac{2 f_{0}^{\prime}}{\pi}\left(y_{0} \log \vartheta+0 x_{0}-y_{0}\right)+\mathrm{E}_{0} y_{0}+\mathrm{J}_{2}+\mathrm{O}\left(\rho^{3} \log \odot\right)\right]-\mathrm{K}_{2}$

Pour $y_{0}=0 \quad \theta=0$, on a $\mathrm{Y}_{2}=0$, d'où $\mathrm{I}_{2}=0, \mathrm{~K}_{2}=0$.

Pour $x_{0}=0 \quad 0=\pi / 2$, on a $\mathrm{X}_{2}=\mathrm{Y}_{1} f_{0}^{\prime} \sin ^{2} k t$.

$K_{1}+\sin ^{2} k t\left[-f_{0}^{\prime}\left(\frac{f_{0}^{\prime}}{\pi}+a_{0}\right) y_{0}+\frac{2 f_{0}^{\prime}}{\pi} y_{0} \log \left|y_{0}\right|-\frac{2 c_{0} f_{0}^{\prime}}{\pi} y_{0}^{2}+\mathrm{I}_{1} \ldots\right]$

$-\frac{a_{0} m_{0}}{2 e \operatorname{sh} m_{0} h} \sin 2 k t\left\lceil-f_{0}^{\prime} y_{0}+\mathrm{J}_{1}+\ldots\right\rceil=\sin ^{2} k t\left[\frac{2 f_{0}^{\prime}{ }^{2}}{\pi} y_{0} \log \left|y_{0}\right|-\left(\frac{2 f_{0}^{\prime}}{\pi}+a_{0}\right) y_{0}+\ldots\right]$ d'où :

$$
\mathrm{I}_{1}=0 \quad \mathrm{~J}_{1}=0 \quad \mathrm{~K}_{1}=0
$$

On connaît donc le développement de $\mathrm{X}_{2}$ et $\mathrm{Y}_{2}$ au voisinage du pied du batteur.

\section{B) Étude du point situé au sommet du batteur}

On transporte à nouveau l'origine des axes au sommet du batteur. On déterminera, comme pour le point situé au pied du batteur, les singularités de la solution particulière du système cidessous en se limitant au cas du batteur plan:

$$
\begin{aligned}
& {\left[f\left(y_{0}\right)=1+\frac{\Delta e}{e h} y_{0}\right]} \\
& \frac{\partial \mathrm{X}_{2}}{\partial x_{0}}+\frac{\partial \mathrm{Y}_{2}}{\partial y_{0}}=\sin ^{2} k t\left\{\begin{array}{l}
\frac{\mathrm{R}^{2}}{g^{2}}\left[(\log \rho)^{2}+\theta^{2}\right]-\frac{2 \mathrm{R}}{g}\left(a_{1}-\frac{\mathrm{R}}{g}\right) \log \rho+\frac{2 \mathrm{R} k^{2} f_{h} \theta}{g^{2}} \\
\left.+\frac{k^{4} f_{h}^{2}}{g^{2}}+\left(a_{1}-\frac{\mathrm{R}}{g}\right)^{2}+O\left[\operatorname{Oog}_{\rho}\right)^{2}\right\rceil
\end{array}\right\} \\
& -\frac{a_{0} m_{0}}{2 e \operatorname{sh} m_{0} h} \sin 2 k t\left\{-\frac{2 \mathrm{R}}{g} \log \rho+2 a_{1}-\frac{2 \mathrm{R}}{g}+O[\rho \log \rho\rceil\right\}+\frac{a_{0}^{2} m_{0}^{2} \cos ^{2} k t}{e^{2} \operatorname{sh}^{2} m_{0} h}\left\lceil 1+O\left(\varrho^{2}\right)\right\rceil \\
& \frac{\partial X_{2}}{\partial y_{0}}-\frac{\partial Y_{2}}{\partial x_{0}}=0
\end{aligned}
$$

d'où la solution particulière :

$$
\begin{aligned}
& \mathrm{X}_{2}^{\prime}=\sin ^{2} k t\left\{\begin{array}{l}
\frac{\mathrm{R}^{2}}{2 g^{2}}\left[x_{0}(\log \varrho)^{2}-x_{0} \log \rho+x_{0} \theta^{2}-y_{0} \theta\right]+\frac{\mathrm{R}}{2 g}\left(\frac{\mathrm{R}}{g}-a_{1}\right)\left(2 x_{0} \log \rho-x_{0}\right) \\
+\frac{\mathrm{R} k^{2} f_{h}}{2 g^{2}}\left(2 \theta x_{0}-y_{0}\right)+o\left[\rho^{2}(\log \rho)^{2}\right]+\left[\frac{k^{4} f_{h}^{2}}{g^{2}}+\left(\frac{\mathrm{R}}{g}-a_{1}\right)^{2}\right] x_{0}
\end{array}\right. \\
& \left.+\frac{a_{0} m_{0}}{2 e \operatorname{sh} m_{0} h} \sin 2 k t\right) \frac{\mathrm{R}}{2 g}\left(2 x_{0} \log \rho-x_{0}\right)+2\left(\frac{\mathrm{R}}{g}-a_{1}\right) x_{0}+\mathrm{O}\left(\rho^{2} \log \rho\right) \\
& +\frac{a_{0}^{2} m_{0}^{2} \cos ^{2} k t}{e^{2} \operatorname{sh}^{2} m_{0} h}\left\lceil x_{0}+\mathrm{O}\left(\rho^{3}\right)\right\rceil
\end{aligned}
$$




$$
\begin{aligned}
& \mathrm{Y}_{22}^{\prime}=\sin { }^{2} k t\left\{\begin{array}{l}
\frac{\mathrm{R}^{2}}{2 g^{2}}\left[y_{0}(\log \rho)^{2}-y_{0} \log \rho+y_{0} \theta^{2}+x_{0} \theta\right]+\frac{\mathrm{R}}{2 g}\left(\frac{\mathrm{R}}{g}-a_{1}\right)\left(2 y_{0} \log \rho-y_{0}\right) \\
+\frac{\mathrm{R} k^{2} f_{h}}{2 g^{2}}\left(2 g y_{0}+x_{0}\right)+\mathrm{O}\left[\rho^{2}(\log \rho)^{2}\right]+\left[\frac{k^{4} f_{h}^{2}}{g^{2}}+\left(\frac{\mathrm{R}}{g}-a_{1}\right)^{2}\right] y_{0}
\end{array}\right. \\
& +\frac{a_{0} m_{0} \sin 2 k t}{2 e \operatorname{sh} m_{0} h}\left\{\frac{\mathrm{R}}{2 g}\left(2 y_{0} \log \rho-y_{0}\right)+2\left(\frac{\mathrm{R}}{g}-a_{1}\right) y_{0}+\mathrm{O}\left(\rho^{2} \log \rho\right)\right\} \\
& +\frac{a_{0}^{2} m_{0}^{2} \cos ^{2} k t}{e^{2} \operatorname{sh}^{2} m_{0} h}\left\lceil y_{0}+\mathrm{O}\left(\rho^{3}\right)\right\rceil
\end{aligned}
$$

Lorsqu'on approche du sommet du batteur en suivant l'axe $O x_{0}$ (surface libre), la partie réelle de la fonction analytique $\mathrm{F}\left(z_{0}\right)=\mathrm{X}_{2}^{\prime \prime}-i \mathrm{Y}_{2}^{\prime \prime}$ vérifie la condition :

$-k^{2} \mathrm{X}_{2}^{\prime \prime}+g \frac{\partial \mathrm{X}_{2}^{\prime \prime}}{\partial y_{0}}=\left(k^{2} \mathrm{X}_{2}^{\prime}-g \frac{\partial \mathrm{X}_{2}^{\prime}}{\partial y_{0}}\right)_{y_{0}=0}+k^{2}\left(\mathrm{X}_{1} \frac{\partial \mathrm{X}_{1}}{\partial x_{0}}+\mathrm{Y}_{1} \frac{\partial \mathrm{Y}_{1}}{\partial x_{0}}\right)_{y_{0}=0} \sin ^{2} k t+\frac{a_{0} m_{0} k^{2}}{2 e^{2} \operatorname{sh}^{2} m_{0} h} \sin 2 m_{0} x_{0} \cos ^{2} k t$ $-\frac{a_{0} m_{0}^{2} g}{2 e} \sin 2 k t\left[\mathrm{X}_{1} \cos m_{0} x_{0}+\frac{k^{2}}{m_{0} g} \mathrm{Y}_{1} \sin m_{0} x_{0}\right] y_{0}=0$

$$
+\frac{a_{0} m_{0} g}{2 e} \sin 2 k t\left[-\frac{\partial \mathrm{X}_{1}}{\partial x_{0}} \sin m_{0} x_{0}+\frac{k^{2}}{m_{0} g} \frac{\partial \mathrm{Y}_{1}}{\partial x_{0}} \cos m_{0} x_{0}\right] y_{0}=0
$$

Lorsqu'on approche du point singulier en suivant l'axe oy (position de repos du batteur), on a au contraire :

$$
\begin{aligned}
& -k^{2} \mathrm{X}_{2}^{\prime \prime}+g \frac{\partial \mathrm{X}_{2}^{\prime \prime}}{\partial y_{0}}=\left(k^{2} \mathrm{X}_{2}^{\prime}-g \frac{\partial \mathrm{X}_{2}^{\prime}}{\partial y_{0}}\right)_{x_{0}=0}-\frac{a_{0}}{e^{2}} \frac{\Delta e}{h} \frac{\sin 2 k t}{\operatorname{sh} m_{0} h}\left[m_{0} g \operatorname{ch} m_{0}\left(y_{0}+h\right)-k^{2} \operatorname{sh} m_{0}\left(y_{0}+h\right)\right] \\
& +\left[-k^{2} \frac{\Delta e}{h e} \mathbf{Y}_{1}+g \frac{\Delta e}{h e} \frac{\partial \mathbf{Y}_{1}}{\partial y_{0}}\right]_{0}=\sin ^{2} k t
\end{aligned}
$$

En remplacant $X_{2}^{\prime}, X_{1}, Y_{1}$ par leurs développements limités, on peut expliciter ces deux conditions limites et déterminer les valeurs de $-k^{2} \mathrm{X}_{2}^{\prime \prime}+g\left(\partial \mathrm{X}_{2}^{\prime \prime} / \partial y_{0}\right)$ à l'origine.

On trouve ainsi :

$1^{\circ} \quad-k^{2} \mathrm{X}_{2 i}^{\prime \prime}+g \frac{\partial \mathrm{X}_{2}^{\prime \prime}}{\partial y_{0}}=\left\{-\frac{k^{2} \mathrm{R} f_{h}}{g} \log \left|x_{0}\right|-k^{2}\left[\frac{3 \mathrm{R} f_{h}}{2 g}-a_{1} f_{h}+\frac{b_{0} k^{2} f_{h}}{g}\right\rceil\right\} \sin ^{2} k t$

$+\frac{a_{0} f_{h}}{2 e g}\left[k^{4}-m_{0}^{2} g^{2}\right] \sin 2 k t+\left\{O\left[x_{0}\left(\log x_{0}\right)^{2}\right]\right\} \sin ^{2} k t$

$+\left\{O\left\lceil x_{0} \log x_{0}\right\rceil\right\} \sin 2 k t+\left\lceil O\left(x_{0}\right)\right\rceil \cos ^{2} k t$

$$
\begin{aligned}
& 2^{\circ}-k^{2} \mathrm{X}_{2}^{\prime \prime}+g \frac{\partial \mathrm{X}_{2}^{\prime \prime}}{\partial y_{0}}=\left\{\mathrm{R} f_{h}^{\prime} \log \left|y_{0}\right|+\left(\mathrm{R} f_{h}^{\prime}+\mathrm{R} \frac{k^{2} f_{h}}{2 g}-\frac{\pi \mathrm{R}^{2}}{4 g}-a_{1} g f_{h}^{\prime}+k^{2} b_{0} f_{h}^{\prime}\right)\right\} \sin ^{2} k t \\
& \left.+\frac{a_{0} f_{h}^{\prime}}{2 e k^{2}}\left[k^{4}-m_{0}^{2} g^{2}\right] \sin 2 k t+\left\{O\left[y_{0} \log y_{0}\right)^{2}\right]\right\} \sin ^{2} k t \\
& \left.+\left\{\mathrm{O}\left[y_{0} \log y_{0}\right)\right]\right\} \sin 2 k t+\mathrm{O}\left(y_{0}\right) \cos ^{2} k t
\end{aligned}
$$

Le sommet du batteur est ainsi un point critique de la fonction :

$$
-k^{2} \mathrm{~F}\left(z_{0}\right)+g i\left[d \mathrm{~F}\left(z_{0}\right) / d z_{0}\right]
$$

Au voisinage de ce point, elle se comporte comme: où l’on a posé :

$-i \mathrm{~A}\left[\log \left(z_{0} e^{i \alpha}\right)\right]^{2} \sin ^{2} k t-i \mathrm{~B} \log \left(z_{0} e^{i \beta}\right) \sin ^{2} k t-i \mathrm{C} \log \left(z_{0} e^{i \gamma}\right) \sin 2 k t$

$$
\mathrm{A}=-\frac{\mathrm{R}\left(k^{2} f_{h}+g f_{h}^{\prime}\right)}{g \pi} \quad x=\frac{\pi k^{2} f_{h}}{2\left(k^{2} f_{h}+g f_{h l}^{\prime}\right)}
$$




$$
\begin{gathered}
\beta=\frac{f_{h} k^{2} \pi\left[3 \mathrm{R}-2 a_{1} g+2 k^{2} b_{0}\right]}{\left.\left(k^{2} f_{h}+g f_{h}^{\prime}\right)\left(4 a_{1} g\right)+k^{2} b_{0}+4 \mathrm{R}\right)+4 \mathrm{R} k^{2} f_{h}-\pi \mathrm{R}^{2}} \\
\mathrm{~B}=-\frac{\left(k^{2} f_{h}+g f_{h}^{\prime}\right)\left(4 a_{1} g+k^{2} b_{0}+4 \mathrm{R}\right)+4 \mathrm{R} k^{2} f_{h}-\pi \mathrm{R}^{2}}{2 \pi g} \\
\mathrm{C}=\frac{\mathrm{R} a_{0}}{2 e g k^{2}}\left(k^{4}-m_{0}^{2} g^{2}\right) \quad \gamma=\frac{k^{2} f_{h}}{\mathrm{R}}
\end{gathered}
$$

On a donc, en intégrant, et en développant $e^{-\left(i k^{2} / g\right) \tilde{w}_{0}}$ :

$$
\mathrm{F}\left(z_{0}\right)=\mathrm{K}\left(z_{0}\right) e^{-\left(i k^{2} / g\right) z_{0}}
$$

$g i \frac{d \mathrm{~K}}{d z_{0}}=e^{\left(i k^{2} / g\right) \tilde{*}_{0}}\left[-i \mathrm{~A}\left(\log z_{0} e^{i(i) 2} \sin ^{2} k t-i \mathrm{~B}\left(\log z_{0} e^{i \beta}\right) \sin ^{2} k t-i \mathrm{C}\left(\log z_{0} e^{i \gamma}\right) \sin 2 k t\right]+\ldots\right.$

$$
\begin{aligned}
& \mathrm{F}\left(z_{0}\right)=\mathrm{K}-\frac{\mathrm{A}}{g} \sin ^{2} k t\left\{z_{0}\left(\log z_{0}\right)^{2}+2(i x-1) z_{0} \log z_{0}+\mathrm{O}\left[z_{0}{ }^{2}\left(\log z_{0}\right)^{2}\right\rceil\right\} \\
& -\frac{\mathrm{B}}{g} \sin ^{2} k t\left[z_{0} \log z_{0}+\mathrm{O}\left(z_{0}^{2} \log z_{0}\right)\right]-\frac{\mathrm{C}}{g} \sin 2 k t\left[z_{0} \log z_{0}+\mathrm{O}\left(z_{0}^{2} \log z_{0}\right)\right]
\end{aligned}
$$

d'où :

$$
\begin{aligned}
& \mathrm{X}_{2}^{\prime \prime}=\mathrm{K}_{1}-\frac{\mathrm{A}}{g} \sin ^{2} k t\left\{x_{0}\left[(\log \rho)^{2}-\theta^{2}\right]-2 y_{0} \theta \log \rho-2\left(x_{0} \log \rho\right)-2 \alpha\left(y_{0} \log \rho+0 x_{0}\right)\right\} \\
& \left.-\frac{\mathrm{B}}{g} \sin ^{2} k t\left\{x_{0} \log \rho-0 y_{0}\right\}-\frac{\mathrm{C}}{g} \sin 2 k t ; x_{0} \log \rho-0 y_{0}\right\}+\ldots \\
& \mathrm{Y}_{2}^{\prime \prime}=\mathrm{K}_{2}-\frac{\mathrm{A}}{g} \sin ^{2} k t\left\{y_{0}\left[(\log \rho)^{2}-0^{2}\right]+2 x_{0} 0 \log \varphi+2 \alpha\left[x_{0} \log \rho-0 y_{0}\right]-2\left(y_{0} \log \varphi+0 x_{0}\right)\right\} \\
& \left.\left.-\frac{\mathrm{B}}{g} \sin ^{2} k t\left\{y_{0} \log \rho+\theta x_{0}\right\}-\frac{\mathrm{C}}{g} \sin 2 k t\right\} y_{0} \log \rho+\theta x_{0}\right\}
\end{aligned}
$$

Les fonctions $\mathrm{X}_{2}^{\prime \prime}\left(y_{0}\right)$ et $\mathrm{Y}^{\prime \prime}{ }_{2}\left(y_{0}\right)$ sont encore de carré intégrable sur l'intervalle $0, h$. Elles sont d'ailleurs finies et continues aussi bien au sommet qu'au pied du batteur.

\section{BIBLIOGRAPHIE}

Havelocr. - - Philosophical Magazine. Série 7, vol. 8, $\mathrm{n}^{\circ} 51$ (oct. 1929).

Biesel (F.). - - La Houllle Blanche, 1951, $\mathrm{n}^{\circ} 2$, p. 156 à $165 ; \mathrm{n}^{\circ} 4$, p. 475 à 496 et mai-juin 1952, p. 372 à 375 .

Kravtchenko (J.). - Coastal Engineering, 5* Conférence, Grenoble, p. 50 à 61 et 189 à 207.

Mrche (R.). - Annales des Ponts et Chaussées, 1944.

Chabert D'Hières (G.). - Extraits des comptes rendus des séances de l'Académie des Sciences, 244, p. 2474-2476, 13 mai 1957.

Apté (A. - Publications scientifiques du Ministère de l'Air, $\mathrm{n}^{\circ} 333$.

Daubert (A.). - Thèse de Doctorat de spécialité. Faculté des Sciences de Grenoble, juin 1958 .

Kravtchenko (J.) et Santon (L.). - Congrès A.I.R.H., juillet 1957, Lisbonne.

Santon (L.). - Coastal Engineering, 5: Conférence, Grenoble, p. 189 à 207. 
PLA N CHE I

\section{CALCUL NUMERIQUE DE L'HARMONIQUE}

On a posé :

$$
\begin{gathered}
\mathrm{B}=\frac{l k^{2} h}{g}=m_{0} h \text { th } m_{0} h \\
\mathrm{~A}_{0}=\frac{a_{0}}{e} \quad \mathrm{~A}_{p}=\frac{a_{p}}{e} \quad \mathrm{M}_{r}=\mu_{r} h \quad \mathrm{~L}_{p}=\lambda_{p} h \quad \mathrm{~L}_{0}=m_{0} h \quad \mathrm{~L}_{0}^{\prime}=m_{0}^{\prime} h
\end{gathered}
$$

On a ainsi :

$$
\begin{aligned}
& \frac{A_{0}}{\operatorname{sh} \mathrm{L}_{0}}=\frac{2 \operatorname{sh} \mathrm{L}_{0}}{\mathrm{~L}_{0}+\operatorname{sh~} \mathrm{L}_{0} \operatorname{ch~} \mathrm{L}_{0}}+\frac{2 \Delta e}{e} \frac{\mathrm{L}_{0} \operatorname{sh~} \mathrm{L}_{0}-\operatorname{ch~} \mathrm{L}_{0}+1}{\mathrm{~L}_{0}\left(\mathrm{~L}_{0}+\operatorname{sh~} \mathrm{L}_{0} \operatorname{ch~} \mathrm{L}_{0}\right)} \\
& \frac{2 \mathrm{~A}_{p}}{\sin \mathrm{L}_{p}}=\frac{2 \sin \mathrm{L}_{p}}{\mathrm{~L}_{p}+\sin \mathrm{L}_{p} \cos \mathrm{L}_{p}}+\frac{2 \Delta e}{e} \frac{\mathrm{L}_{p} \sin \mathrm{L}_{p}+\cos \mathrm{L}_{p}-1}{\mathrm{~L}_{p}\left(\mathrm{~L}_{p}+\sin \mathrm{L}_{p} \cos \mathrm{L}_{p}\right)} \\
& \frac{h c_{r}^{\prime}}{e^{2}}=\frac{2}{\pi(1+r)}\left\{\begin{array}{l}
+\frac{\Delta e}{e} \sum_{p=1}^{\infty} \frac{\mathrm{A}_{p} \mathrm{~L}_{p}}{\left(\mathrm{M}_{r}{ }^{2}-\mathrm{L}_{p}{ }^{2}\right) \sin \mathrm{L}_{p}}\left[(-1)^{r} \cos \mathrm{L}_{p}+1\right] \\
+(-1)^{r} \frac{\mathrm{A}_{0} \mathrm{~L}_{0}}{\mathrm{~B}} \sum_{p=1}^{p=-\infty} \frac{\mathrm{A}_{p}\left(\mathrm{~B}^{2}-\mathrm{L}_{p}{ }^{2}\right)\left(\mathrm{L}_{0}{ }^{2}+\mathrm{L}_{p}{ }^{2}+\mathrm{M}_{r}{ }^{2}\right)}{\left(\mathrm{L}_{0}{ }^{2}-\mathrm{L}_{p}{ }^{2}+\mathrm{M}_{r}{ }^{2}\right)^{2}+4 \mathrm{~L}_{0}{ }^{2} \mathrm{~L}_{p}{ }^{2}} \\
-\frac{(-1)^{r}}{\mathrm{~B}} \sum_{p=1}^{p=\infty} \sum_{q=1}^{\infty} \mathrm{A}_{p} \mathrm{~A}_{q}\left(\mathrm{~L}_{p}+\mathrm{L}_{q}\right) \frac{\mathrm{M}_{r}{ }^{2}\left(\mathrm{~B}^{2}-\mathrm{L}_{p} \mathrm{~L}_{q}\right)-\left(\mathrm{L}_{p}-\mathrm{L}_{q}\right)^{2}\left(\mathrm{~B}^{2}+\mathrm{L}_{p} \mathrm{~L}_{q}\right)}{\left(\mathrm{L}_{p}{ }^{2}+\mathrm{L}_{q}{ }^{2}-\mathrm{M}_{r}{ }^{2}\right)^{2}-4 \mathrm{~L}_{p}{ }^{2} \mathrm{~L}_{q}{ }^{2}}
\end{array}\right.
\end{aligned}
$$

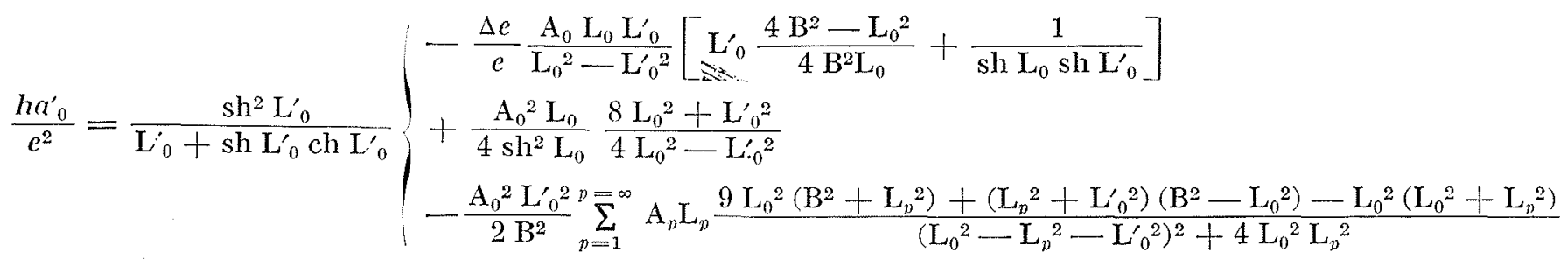

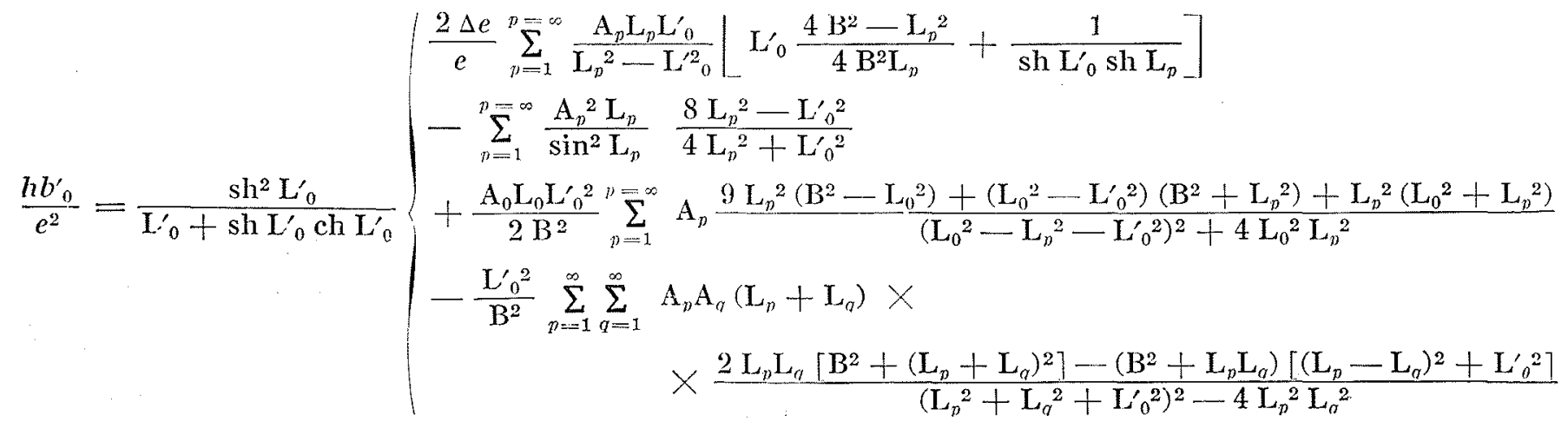

On peut calculer également :

$$
\frac{\mathrm{T}^{2} a_{0}^{\prime} g}{a_{0}^{2}}=\left(\frac{h \alpha_{0}^{\prime}}{e^{2}}\right) \times \frac{4 \pi^{2}}{\mathrm{BA}_{0}{ }^{2}} \quad \frac{\mathrm{T}^{2} b_{0}^{\prime} g}{a_{0}^{2}}=\left(\frac{h b_{0}^{\prime}}{e^{2}}\right) \times \frac{4 \pi^{2}}{\mathrm{BA}_{0}{ }^{2}}
$$

ou encore:

$$
\frac{\mathrm{T}^{2} a_{0}^{\prime} g}{e^{2}}=\left(\frac{h^{\prime} a_{0}}{e^{2}}\right) \times \frac{4 \pi^{2}}{\mathrm{~B}} \quad \frac{\mathrm{T}^{2} b_{0}^{\prime} g}{e^{2}}=\left(\frac{h b_{0}^{\prime}}{e^{2}}\right) \times \frac{4 \pi^{2}}{\mathrm{~B}}
$$


P L A N CHE II

\section{ABAQUES DE L'HARMONIQUE EMIS PAR UN BATTEUR-PISTON}

(Théorie au $2^{\circ}$ ordre)

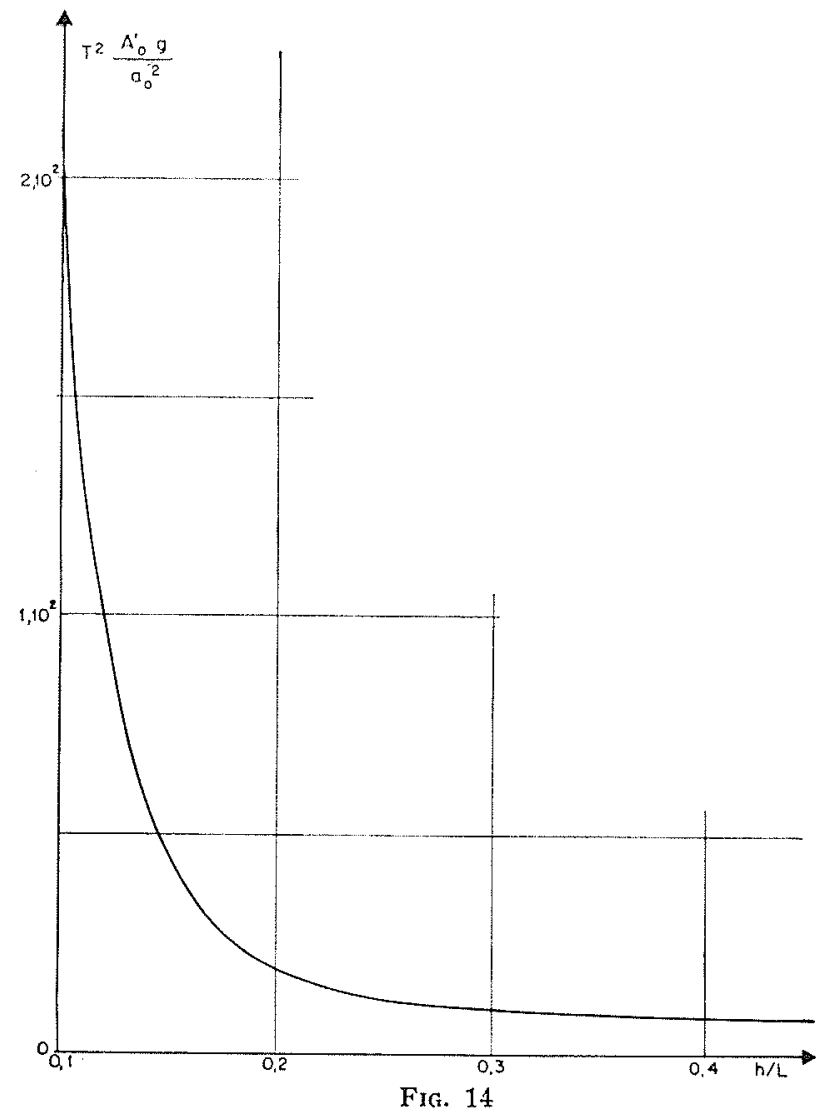

Abaque donnant la demi-amplitude de l'harmonique linéaire

$\frac{\mathrm{T}^{2} \mathrm{~A}_{0}^{\prime} g}{a_{0}^{2}}=\frac{\mathrm{T}^{2} \sqrt{a_{0}{ }^{2}+b_{0}{ }^{\prime 2}} g}{a_{0}{ }^{2}}$

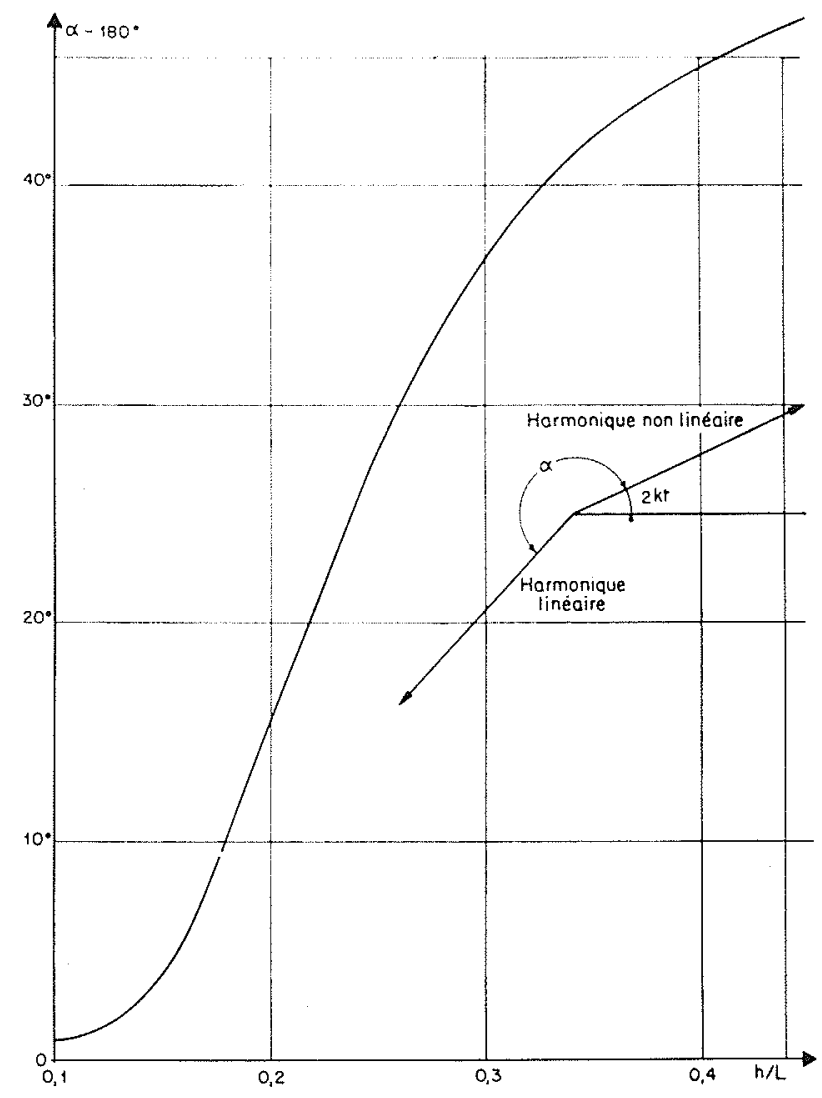

FIG. 15

Abaque donnant la phase de l'harmonique linéaire

$\alpha-180^{\circ}=\operatorname{arctg}\left(b_{0}^{\prime} / a_{0}^{\prime}\right)$ 